\title{
Les relations plantes-insectes phytophages chez les femelles pondeuses : le rôle des stimulus chi- miques et physiques. Une mise au point biblio- graphique
}

Pierre-Charles ROBERT

I.N.R.A., Station de Zoologie, 28, rue de Herrlisheim, F-68021 Colmar

Mots clés additionnels : Kairomone, allomone, phéromone, stimulus optique, polymorphisme de la ponte. and physical stimulus. $A$ review.

This paper reviews the present knowledge concerning the role played by chemical and physical signals in host identification by egg-laying females of phytophagous insects. The past 15 years' research has brought original results in fields like the physiology, ethology, ecology of insects and agronomy : confirmation of the action of plant kairomones in specific host-finding and in egg-laying stimulation; the discovery of the action of nonhost-plants but also of host-plant allomones; significance of the optical properties of plants ; the close association between physical stimuli and chemical signals at each step of the egg-laying behaviour; chemical and visual mechanisms utilized to prevent repeated egg-laying in a single host and to avoid intra-specific, and sometimes inter-specific, competition; different expressions of polymorphism in egg-laying behaviour ; stimulation of ovarian activity by means of host kairomones. The applications in agriculture are discussed.

Additional key words : Kairomone, allomone, pheromone, optical stimulus, polymorphism in egg-laying behaviour.

\section{INTRODUCTION}

Le choix de la plante-hôte par une femelle au moment de la ponte est déterminant pour le succès de la descendance, particulièrement chez les espèces qui ont des stades larvaires jeunes peu mobiles. Lorsque adultes et jeunes se nourrissent du même végétal, la sélection du site de ponte peut être conditionnée par le comportement de prise alimentaire des parents. Mais chez beaucoup d'espèces, les adultes ne s'alimentent pas ou utilisent une alimentation différente de celle de leur progéniture; les femelles gravides sélectionnent 
cependant le végétal-hôte des descendants avec une précision qui a étonné les premiers observateurs qui ont parlé d'《instinct botanique » (PESSON, 1980). Nous envisageons, dans cet exposé, de faire une revue de nos connaissances sur les mécanismes qui président au comportement du choix des pondeuses surtout de cette dernière catégorie pour laquelle les facteurs d'ordre alimentaire semblent exclus.

L'existence de liens physiques et chimiques entre insectes et plantes a été reconnue dès le début du $\mathrm{XX}^{\mathrm{e}}$ siècle (VERSCHÄFFELT, 1910 ; RICHARDSON, 1925). Les études intensives sur les relations plantesinsectes ont commencé après 1950 d'abord sur le comportement alimentaire (THORSTEINSON, 1960 ; BECK, 1965 ; FRAENKEL, 1959, 1969 ; SCHOONHOVEN, 1968, 1981, 1982). Les travaux sur la ponte ont évolué plus lentement de sorte que KOGAN (1976) écrivait : «Particularly deficient is our knowledge on host-finding by ovipositing females and the chemical regulation of oviposition selection $»$. Depuis cette date, un nombre considérable de recherches ont comblé cette lacune. Leurs motivations concernent la science fondamentale dans des disciplines diverses : l'éthologie, la physiologie sensorielle, la physiologie de la reproduction, l'écologie, la chimie des substances naturelles, mais aussi l'entomologie appliquée. C'est sous ce dernier aspect que sont exposées ici les relations plantesinsectes pendant la ponte.

Nous étudierons successivement la perception des plantes-hôtes et non-hôtes, la perception de congénères sur les plantes-hôtes, le polymorphisme des réactions des femelles, la stimulation de l'ovogenèse par les plantes-hôtes et enfin les applications possibles en entomologie agricole.

\section{LA PERCEPTION DES VÉGÉTAUX LORS DU COMPORTEMENT DE PONTE DES INSECTES PHYTOPHAGES}

Une femelle pondeuse répond à des stimulus physiques et chimiques quand elle choisit dans son habitat une plante support de ponte. Les stimulus chimiques fabriqués par le végétal et par l'animal sont complexes et agissent par olfaction et gustation. Une terminologie a été établie selon leur origine et le mode d'action ; nous utilisons ici celle proposée par NORDLUND (1981).

\section{A. La perception des plantes-hôtes}

\section{Le choix d'une famille ou d'une espèce de plante}

Le dépôt réussi des œufs dépend de séquences comportementales liées à la présence d'une ou de plusieurs espèces de plantes nourricières des larves. Trois étapes principales peuvent être distinguées : $a$ ) la découverte à distance de la plante-hôte, $b$ ) l'identification rapprochée et/ou au contact de l'hôte, c) l'émission des œufs. L'étude du comportement de 2 ravageurs des crucifères, la piéride du chou Pieris brassicae L. (Lepid. Pieridae) et la mouche du chou Delia radicum L. = Hylemia brassicae Bouché (Dipt. Hylemyiidae), met en lumière le rôle de différents stimulus. Les crucifères sont caractérisées par la présence de glucosinolates non volatils qui, par hydrolyse enzymatique, dégagent des composés volatils : isothiocyanates, thiocyanates, nitriles (FINCH, 1977 ; LERIN, 1980).

$\mathrm{Au}$ départ, les femelles gravides de la piéride du chou sont attirées vers les surfaces vertes (ILSE, 1937) et peut-être aussi par les odeurs des crucifères (SCHÜTTE, 1966). Mais les odeurs semblent plutôt fonctionner comme « arrestant » (MITCHELL, 1977) et les femelles se concentrent dans des zones étroites où des crucifères sont présentes. Là, elles se posent sur les différents végétaux ; sur les non-hôtes, elles tambourinent brièvement avec les tarses antérieurs sur la face supérieure des feuilles et s'envolent ; après la rencontre avec une crucifère, le tambourinage est réalisé à grande vitesse, puis la ponte commence à la face inférieure d'une feuille (TEROFAL, 1965). L'émission des œufs n'est pas commandée par les composés volatils, elle est une réponse aux stimulus chimiques de contact perçus au niveau des tarses (MA, 1969 ; MA \& SCHOONHOVEN, 1973). Les œufs sont déposés sur une surface lisse d'une feuille détectée par des récepteurs tactiles de l'ovipositeur.

Au début de leur quête, les pondeuses de la mouche du chou s'engagent dans un vol spontané contre le vent sans être influencées par la présence de crucifères. Les odeurs typiques sont perçues seulement lorsque les femelles arrivent à une distance allant de 5 à $10 \mathrm{~m}$ des hôtes (FINCH \& SKINNER, 1982b) ; ces kairomones stimulent l'activité des mouches qui, en se déplaçant toujours contre le vent, se rapprochent alors rapidement des plantes-hôtes (TRAYNIER, $1967 a$; FINCH, 1978 ; STÄDLER, 1978 ; HAWKES \& CoAKer, 1979 ; Walbank \& Weatley, 1979). Puis, dans le voisinage immédiat d'une crucifère, à environ $25 \mathrm{~cm}$, le choix du site d'atterrissage est entièrement visuel ; les hôtes sont détectés d'après la couleur et la taille des feuilles (PROKOPY et al., 1983a et $b$ ). Après atterrissage, la plante est reconnue par la perception des glucosinolates au niveau de récepteurs de contact des tarses qui n'existent pas chez les mâles (STÄDLER, 1978). Ensuite les femelles, stimulées par les kairomones de contact, se déplacent sur les feuilles puis descendent sur le sol à la base de la tige. Les œufs ne sont pas déposés sur la plante, mais à proximité de celle-ci, dans le sol s'il présente des qualités granuleuses particulières perçues tactilement au niveau de l'ovipositeur (TRAYNIER, 1967b; ZOHREN, 1968). L'émission des œufs est induite par les glucosinolates, les composés volatils des crucifères jouent alors un rôle de synergie (NAIR \& MC EWEN, 1976 ; STÄDLER, 1978).

Ces 2 exemples mettent en relief la participation de 4 fonctions sensorielles : la vue, le toucher, l'olfaction, la gustation. C'est presque toujours une imbrication de différents stimulus physiques et chimiques qui intervient et qui guide les différentes étapes de l'activité de ponte depuis l'approche jusqu'à l'émission des œufs.

Les comportements de ces 2 insectes déprédateurs des crucifères sont très différents : la piéride pond sur une feuille lisse, la mouche dans un sol granuleux. Les odeurs n'influencent pas la ponte de la première, tandis qu'elles stimulent celle de la deuxième. La vue a un grand rôle dans la reconnaissance de la plante chez la mouche, mais pas chez la piéride. Une variabilité de réactions existe donc entre espèces dans le choix du site de ponte et dans le déterminisme de l'émission des 
œufs. Aussi en agronomie, il est indispensable de réaliser des études particulières pour chaque ravageur. Des analyses importantes sont déjà faites pour certaines espèces. Citons parmi les ravageurs européens : la piéride de la rave, Pieris rapae L. (Lepid. Pieridae) (TEROFAL, 1965 ; IVES, 1978 ; TRAYNIER, 1979 ; JONES \& IVES, 1979 ; RENWICK \& RADKE, 1983) ; la teigne du poireau, Acrolepiopsis assectella Zell. (Lepid. Plutellidae) (AUGER \& THIBOUT, 1981, 1983 ; THIBOUT \& AUGER, 1983 ; THIBOUT et al., 1982, 1985) ; la teigne de la betterave, Scrobipalpa ocellatella Boyd. (Lepid. Gelechiidae) (ROBERT, 1965, 1970, 1971, 1976 ; ROBERT \& BLAISINGER, 1978 ; ROBERT et al., 1977) ; la bruche du haricot, Acanthoscelides obtectus Say. (Coleopt. Bruchidae) (LABEYRIE, 1961 ; POUZAT, 1970, 1976, 1978, 1981) ; de nombreux diptères Tephritidae: la mouche de l'olive, Dacus oleae Gmel. (PROKOPY \& HANIOTAKIS, 1975, 1976 ; HANIOTAKIS \& VOYADJOGLOU, 1978 ; GIROLAMI et al., 1981, 1983 ; KATSOYANNOS \& PITTARA, 1983 ; LEVINSON \& LEVINSON, 1984) ; la mouche des cerises, Rhagoletis cerasi L. (PROKOPY \& BOLLER, 1971 ; HURTER et al., 1976 ; HAISCH \& LEVINSON, 1980 ; REMUND et al., 1980 ; LEVINSON \& HAISCH, 1984) ; la mouche des fruits, Ceratitis capitata Wied. (FERON, 1962; SANDERS, 1962, 1968 ; PROKOPY et al., 1978) ; la mouche de la carotte, Psila rosae F., (Dipt. Psilidae) (STÄDLER, 1972, 1977 ; BRUNEL, 1977 ; GUERIN, 1980 ; STÄDLER \& BUSER, 1982 ; GUERIN \& STÄDLER, 1982 ; GUERIN et al., 1983); la mouche de l'oignon, Hylemya antiqua Meig. (Dipt. Anthomyiidae) (VERNON et al., 1977, 1978 ; PIERCE et al., 1978 ; ISHIKAWA et al., 1978, 1981 ; Dindonis \& Miller, $1980 a$; HARRIS \& MILLER, 1982, 1983).

\section{Le choix d'individus particuliers dans une espèce végétale}

Les comportements rapportés jusqu'ici conduisent au choix d'hôtes appartenant à une espèce ou à quelques espèces végétales dans une communauté de plantes. Dans certains cas des pondeuses réalisent un $2^{\mathrm{e}}$ type de choix en préférant certains individus à l'intérieur d'une espèce végétale et, en agriculture, certains cultivars. Il en résulte une répartition contagieuse des pontes; des pieds attirent et retiennent les femelles de façon répétitive, alors que d'autres sont régulièrement évités. La taille, la silhouette, la couleur, le contraste entrent en jeu (IVES, 1978 ; LATHEEF \& IRWIN, 1979, RAUSHER et al., 1981 ; COURTNEY, 1982). Cactoblastis cactorum Berg. (Lepid. Phycitidae) choisit les plants de cactus les plus verts et les plus grands, présentant une bonne activité de photosynthèse (MYERS et al., 1981). La vue joue donc un rôle important. Le fait que le choix chez Euphydrias editha Boisduval (Lepid. Nymphalidae) est confirmé après atterrissage semble impliquer ensuite l'action de médiateurs chimiques (RAUSHER et al., 1981).

Peu de recherches sont réalisées à ce niveau, mais des corrélations sont apparues : $P$. brassicae adopte les pieds de crucifères qui dégagent la plus grande quantité de substances volatiles (MITCHELL, 1977); $P$. rapae pond de préférence sur les plantes les plus riches en eau (WOLFSON, 1980). Ellis et al. (1982) émettent l'hypothèse que la préférence marquée par la mouche du chou pour certains pieds de radis pourrait traduire des différences dans les microflores associées aux végétaux et donc traduire des différences dans l'émission de composés volatils métabolisés par chaque microflore. L'âge de la plante peut intervenir (IVES, 1978 ; JONES \& IVES, 1979). Pour la mouche du chou, le radis est le plus attractif entre 25 et $35 \mathrm{j}$, période où la teneur en un composé volatil, le 4méthyl thiobut-3-enyl isothio-cyanate est la plus élevée (HARDMAN \& Ellis, 1978 ; Ellis et al., 1979).

La pyrale du maîs Ostrinia nubilalis Hübn. (Lepid. Pyralidae) choisit d'abord les cultivars les plus riches en sucres (DERRIDJ \& FIALA, 1983). Les nutriments interviendraient donc dans la décision finale de la pondeuse selon des mécanismes qui restent à préciser. C'est un phénomène nouveau qui mérite d'être étudié, car il peut exister chez de nombreux insectes.

Certains auteurs (IVES, 1978 ; JONES \& IVES, 1979) suggèrent que les femelles sélectionnent ainsi les pieds les plus favorables au développement des larves. Le choix de $P$. rapae pour les plantes riches en eau semble aller dans ce sens, car les fortes teneurs en eau favorisent la croissance des chenilles de cette espèce (FEENy, 1975). Pour MYERs et al. (1981), C. cactorum sélectionnerait les individus de cactus les meilleurs pour leur descendance; les femelles auraient donc la capacité de reconnaître par des caractères optiques certains individus végétaux qui sont les plus avantageux pour l'espèce.

\section{Le comportement des pondeuses est-il toujours favorable aux larves?}

Nous venons de voir que des femelles pondeuses adoptaient des individus particuliers à l'intérieur d'une espèce végétale et que ce comportement pouvait être favorable aux larves. Les femelles choisissent-elles aussi parmi plusieurs espèces d'hôtes celles qui assurent le meilleur développement de leur descendance ? Cela est réalisé chez Papilio machaon L. (Lepid. Papilionidae) (WIKLUND, 1974, 1981), chez Rabdophaga terminalis H. Loew (Dipt. Cecidomyiidae) (AHMAN, 1984). Mais la règle n'est pas généralisable; $P$. rapae préfère pondre sur chou plutôt que sur radis, mais la survie des larves est moins bonne sur chou (IVES, 1978, JONES \& IVES, 1979). Les pondeuses d'Anthocharis cardamines L. (Lepid. Pieridae) sélectionnent les crucifères portant les inflorescences les plus grandes ; des végétaux peu favorables à la survie des larves comme Barbarea vulgaris $\mathrm{R}$. Br. et Hesperis matronalis L. reçoivent plus d'œufs que des espèces plus avantageuses pour la descendance ; la sélection est visuelle (COURTNEY, 1982). Chez d'autres espèces, des pontes sont aussi observées sur des plantes défavorables au développement des descendants (NEILSON, 1967 ; FRAENKEL, 1969 ; BOLLER \& PROKOPY, 1976 ; CHEW, $1977 a$ et $b$; JACKAI \& SiNGH, 1981 ; COBBINAH et al., 1982). Inversement, des espèces convenables pour les larves ne reçoivent jamais d'œufs (ClARIDGE \& Wilson, 1978 ; JERMY \& SZENTESI, 1978 ; TICHENOR \& SEIGLER, 1980 ; ABE et al., 1981 ; COURTNEY, 1982). Le choix des femelles entre plusieurs espèces d'hôtes n'a donc pas forcément une valeur adaptative pour l'insecte. 


\section{Le rôle des stimulus physiques et chimiques dans la perception des plantes-hôtes}

Le rôle des kairomones et des caractères physiques des végétaux dans les relations plantes-insectes a fait l'objet d'analyses (KOGAN, 1976 ; FINCH, 1980 ; BECK \& SCHOONHOVEN, 1980 ; SCHOONHOVEN, 1983). Nous relevons ici seulement quelques aspects nouveaux ou intéressant l'agriculture.

\section{a) Les stimulus physiques}

Les caractères physiques des plantes ne sont généralement pas jugés suffisamment typiques pour permettre à un insecte d'identifier une plante-hôte, site de ponte.

Pourtant les facteurs optiques jouent un rôle capital. La mouche du chou se guide sur la couleur et la taille des feuilles pour atterrir sur une crucifère. De nombreuses mouches Tephritidae frugivores localisent dans les arbres les fruits, sites de ponte, par la forme, la couleur, la taille (PROKOPY, 1968, 1977 ; PROKOPY \& OWENS, 1983). Des papillons semblent reconnaître les plantes-hôtes par la vue (GILBERT, 1975 ; RAUSHER, 1979 ; WIKLUND, 1982, 1984). Rappelons que le choix d'individus particuliers à l'intérieur d'une espèce végétale se fait sur des critères-visuels. Il paraît donc tout à fait possible que la vue assure l'identification d'une plante, site de ponte. Le rôle des yeux n'est pas forcément terminé avec l'atterrissage sur l'hôte ; il peut encore intervenir pendant l'émission des œufs : ainsi la ponte de la mouche de l'oignon $H$. antiqua est stimulée par la présence d'une tige verticale jaune (HARRIS \& MILLER, 1982, 1983).

Ces relations optiques plante-insecte sont encore trop peu étudiées, car en agriculture leur connaissance doit conduire à construire des pièges plus performants par leur forme et leur couleur ou à créer des variétés non ou peu identifiables par la vue (PrOKOPY \& OWENS, 1983 ; PROKOPY et al., 1983b).

Parce que le dépôt des œufs est associé à un support, les qualités mécaniques de ce dernier (forme, texture, position) exercent une influence. POUZAT (1976) a montré que la bruche du haricot ne pond que si les kairomones de l'hôte sont associées avec des fentes étroites dans le support. Des relations de cet ordre ont été trouvées chez S. ocellatella (ROBERT, 1965), Phthorimaea operculella Zell. (Lepid. Gelechiidae) (FENEMORE, 1980), Protoparce sexta Johan. (Lepid. Sphingidae) (SPARKS, 1973) et de nombreux autres insectes (MEHTA \& SAXENA, 1970).

\section{b) Les stimulus chimiques : les kairomones}

La perception des stimulus chimiques spécifiques assure l'identification de la plante-hôte. La perception par gustation paraît la plus sûre, car elle signale forcément la matérialité du support de ponte (POUZAT, 1980). L'olfaction joue aussi un rôle ; chez divers insectes, les kairomones volatiles sont impliquées dans la découverte du site de ponte à courte et moyenne distance (RÖTTGER, $1979 a$ et $b$; REISSIG et al., 1982 ; SCHOONHOVEN, 1983 ; KAMM \& BUTTERY, 1983 ; GROVE \& BLIGHT, 1983 ; Girolami et al., 1983). Elles interviennent aussi dans la stimulation de la ponte (NAIR \& MC EWEN, 1976 ; STÄDLER, 1978 ; DINDONIS \& Miller, 1980 $a$; HaRRIS \& Miller, 1982 ; Auger \& ThibouT, 1983 ; LeVINSON \& HaISCH, 1984).
Les kairomones végétales sont presque toujours des substances typiques des espèces-hôtes comme les glucosinolates des crucifères et leurs dérivés. Elles sont quelquefois constituées d'un seul composé (FLETCHER \& WATSON, 1974 ; SUTHERLAND et al., 1977 ; SWIFT, 1982), mais le plus souvent c'est un mélange de composés qui est actif. Différents glucosinolates et leurs produits volatils d'hydrolyse attirent et stimulent les insectes des crucifères. La mouche de l'oignon répond à un mélange de composés soufrés naturels ou de synthèse portant la fonction propylthio (VERNON et al., 1977, 1978 ; PIERCE et al., 1978 ; ISHIKAWA et al., 1978, 1981 ; DINDONIS \& MILLER, $1980 a$; HARRIS \& Miller, 1982). Chez la mouche de la carotte, 5 composés interviennent : 2 phénylpropanö̈des (le méthylisoeugenol et le transasarone), 2 furanocoumarines (le bergaptène et la xanthotoxine), 1 polyacétylénique, le falcarindiol (STÄDLER, 1977 ; GUERIN \& STÄDLER, 1982 ; STÄDLER \& BUSER, 1982). Certains composés ne sont pas des substances du végétal, mais des métabolites élaborés par des microorganismes à partir de substances de la plante-hôte (DINDONIS \& MILLER, 1980b ; HOUGH et al., 1981 ; ELLIS et al., 1982).

Enfin les éléments d'un mélange peuvent ne pas être des composants typiques de la plante-hôte ou de sa famille végétale. Ce sont des substances très communes dans le monde végétal. Mais le mélange de plusieurs de ces substances dans des proportions bien précises caractérise la plante-hôte (REISSIG et al., 1982 ; VISSER, 1983 ; GUERIN et al., 1983).

Le rôle des kairomones a été décrit chez de nombreux autres insectes (annexe 1).

L'agronome est intéressé par l'utilisation de ces kairomones que le chimiste sait identifier et synthétiser. Il peut les utiliser en piégeage, ou essayer, par manipulation génétique, de rendre la plante moins attractive ou moins stimulante pour la ponte en modifiant la composition allélochimique (JACKSON et al., 1984).

\section{B. La perception des plantes non-hôtes : les allomones}

Comme pour la prise alimentaire, il a d'abord été admis que seuls les signaux chimiques spécifiques des plantes-hôtes guident et stimulent les femelles gravides. RICHARDSON (1925), DETHIER (1947) et SCHOONHOVEN (1968) ignorent l'existence de mécanismes chimiques de répulsion ou d'inhibition dans la ponte des phytophages. C'est oublier que, dans leur quête, les femelles rencontrent quantité d'espèces végétales nonhôtes, les reconnaissent et ne pondent pas à leur contact. GUPTA \& THORSTEINSON (1960) découvrent, pour la première fois semble-t-il, que la ponte d'un insecte phytophage, la teigne des crucifères, Plutella maculipennis Curt. (Lepid. Plutellidae), est empêchée par la coumarine de la luzerne et un composé inconnu de la tomate. CHAPMAN (1974) indique seulement 2 autres exemples d'inhibition de la ponte.

Des recherches récentes montrent que des allomones d'origine végétale, répulsives ou inhibitrices, sont nombreuses et qu'elles agissent soit par gustation, soit par olfaction.

Des extraits de plantes de différentes espèces ont des actions négatives sur la ponte de la teigne de la betterave, S. ocellatella; les extraits de châtaignier, 
les plus actifs, sont répulsifs dans le choix d'un support de ponte et inhibent totalement la ponte des teignes privées de betterave. Par ailleurs, pulvérisés sur une betterave, ils masquent l'action stimulante de la plante-hôte qui n'est plus reconnue. Au champ, ils diminuent sensiblement le niveau de population (ROBERT, 1971, 1976 ; ROBERT \& BLAISINGER, 1978).

De même, la ponte de $P$. brassicae est empêchée par des extraits de divers végétaux non-hôtes (LUNDGREN, 1975), par des substances organiques et minérales diverses (JERMY \& SZENTESI, 1978). Des extraits de nombreuses plantes inhibent la ponte de 2 noctuelles: Trichoplusia ni Hübn. (RENWICK \& RADKE, 1981) et Anadevidia peponis Fabr. (ICHINOSE \& SASAKI, 1975). Des composés inhibiteurs actifs chez d'autres phytophages (annexe 2) ont été trouvés chez divers végétaux non-hôtes.

Comme dans l'expérience de GUPTA \& THORSTEIN SON (1960), la ponte des femelles de la teigne de la betterave et de la piéride du chou est inhibée lorsque des composés végétaux étrangers sont épandus sur l'hôte et agissent par contact ; ces allomones sont d'origines diverses et de structures chimiques dissemblables. Les recherches sur les récepteurs sensoriels de ces substances chez les femelles sont peu avancées. JERMY \& SZENTESI (1978) suggèrent que les organes sensoriels sont localisés sur les tarses, l'ovipositeur jouant un rôle secondaire.

L'action négative de composés volatils non-hôtes a été révélée par SAXENA \& BASIT (1982a et $b$ ); des odeurs de ricin, de coriandre, de tomate et des émissions de citral diminuent sensiblement la ponte d'une cicadelle Amrasca devastans Dist. sur son hôte : le coton. Ces résultats confortent l'hypothèse que des allomones de plantes non-hôtes sont répulsives ou masquent les kairomones de l'hôte et que des cultures de plusieurs espèces végétales en mélange doivent gêner la colonisation de chaque plante cultivée et entraîner une diminution des niveaux de populations des ravageurs. Des expérimentations apportent des résultats positifs pour des insectes qui ont des liens avec les odeurs de leur plante-hôte: la mouche du chou (COAKER, 1980 ; THEUNISSEN \& DEN OUDEN, 1980 ; TUKAHIRWA \& COAKER, 1982), la mouche de la carotte (UVAH \& COAKER, 1984), la teigne des crucifères (PERRIN \& Phillips, 1978), l'altise du chou, Phyllotreta cruciferae Geoze (Coleopt. : Chrysomelidae) (TAHVANAINEN \& ROOT, 1972; LATHEEF \& ORTIZ, 1984 ; LATHEEF et al., 1984), le doryphore (PANASIUK, 1984). Par contre, la colonisation des crucifères par la piéride de la rave, qui reconnaît les plantes-non-hôtes et les plantes-hôtes par contact après atterrissage (TEROFAL, 1965) n'est pas diminuée dans des cultures en mélange (THEUNISSEN \& DEN OUDEN, 1980 ; LATHEEF \& ORTIZ, $1983 a$ et $b$; MAGUIRE, 1984).

\section{LA PERCEPTION DE CONGÉNÈRES SUR LA PLANTE-HÔTE}

Chez certains phytophages, l'occupation d'un hôte par un ou plusieurs congénères est reconnue par les pondeuses au moyen de stimulus chimiques et optiques qui inhibent la ponte et dispersent les femelles.
La colonisation est maintenue à un niveau optimal pour exploiter l'hôte et éviter la surpopulation.

\section{A. La perception des congénères par des stimulus chimiques}

La présence de congénères est perçue par les femelles à partir de substances soit d'origine végétale, des allomones de la plante-hôte, soit synthétisées par l'insecte adulte ou larve, des phéromones.

\section{Les allomones dans les plantes-hôtes}

Des plantes-hôtes contiennent des substances répulsives ou inhibitrices (STÄDLER, 1972 ; THIBOUT et al., 1982 ; AUGER \& THIBOUT, 1983). Des insectes utilisent ces allomones pour marquer le site de ponte et le protéger contre l'arrivée de nouveaux congénères. L'exemple de la mouche de l'olive, $D$. oleae, est significatif. Dans l'arbre, les femelles détectent visuellement le fruit par sa forme, sa taille et sa couleur ; sur l'olive, des signaux chimiques spécifiques associés à la texture lisse désignent le site de ponte. La femelle fore le tégument et dépose un œuf. Elle aspire ensuite avec son proboscis le jus qui sourd du trou de ponte et le répand sur le fruit. Ce jus contient des composés qui masquent les kairomones de l'olive et inhibent de nouvelles pontes (CIRIO, 1971 ; PROKOPY \& HANIOTAKIS, 1976 ; HANIOTAKIS \& VOYADJOGLOU, 1978). Ces allomones sont perçues par contact au niveau des tarses et par olfaction par les antennes. La protection est efficace pendant le développement de la jeune larve. Ensuite l'occupation du fruit est détectée par des composés volatils exhalés à travers les lésions du tégument occasionnées par les asticots âgés (GIROLAMI et al., 1981). Ce comportement de marquage par les pondeuses est tout à fait remarquable et doit être rapproché de celui d'autres mouches Tephritidae qui marquent leur hôte par une phéromone, processus étudié dans le chapitre suivant.

D'autres Dacus, D. cucurbitae Coquillet (PROKOPY \& KoYAMA, 1982), D. tryoni Frogg, D. jaroisi Tryon (FITT, 1984) ne marquent pas le fruit avec une allomone et ne discernent pas le passage d'une première pondeuse. Mais, comme $D$. oleae, elles reconnaissent, par des émissions volatiles végétales, les fruits habités par des larves.

Des allomones de l'hôte sont aussi dégagées, soit par la lésion de la ponte chez Hoplocampa testudinea Klug. (Hymen. Tenthredinidae) (RoITBERG \& PROKOPY, 1984), soit par des blessures provoquées par les larves chez la pyrale du maïs (SCHURR, 1970; SCHURR \& HOLDAWAY, 1970). D'autres allomones végétales actives sont présentes dans les fèces des larves de Trichoplusia ni (RENWICK \& RADKE, 1981, 1982). Chez l'anthonome du coton: Anthonomus grandis Boh. (Coleopt. Curculionidae) (STANSLY \& CATE, 1984) et chez la pyrale du maïs (DITTRICK et al., 1983) des substances inhibant la ponte sont présentes dans les fèces des larves, sans que l'on sache si ce sont des allomones ou des phéromones. Cette dernière observation montre que plusieurs mécanismes empêchant la ponte coexisteraient chez certaines espèces comme la pyrale du maïs. 


\section{Les phéromones}

Des femelles d'espèces phytophages émettent une phéromone de marquage qui signale aux congénères la présence d'un œuf comme le font aussi certains entomophages (VAN LENTEREN, 1981) : le phénomène a été découvert pour la première fois chez la mouche de la pomme, Rhagoletis pomonella (Dipt. Tephritidae) par PROKOPY et son école (PROKOPY, 1972, $1981 a$ et $b$ ). Les faits sont assez exemplaires pour être rapportés ici. L'hôte d'origine est le fruit de Crataegus qui assure le développement d'un seul asticot. Après la ponte, la femelle, au cours d'une danse, frotte l'oviscapte sur le fruit et dépose une phéromone qui inhibe la ponte et éloigne les congénères. La durée d'action est de quelques jours pendant lesquels la larve du $1^{\text {er }}$ âge se développe, ensuite les femelles comme celles de $D$. oleae, détectent la présence des larves des $2^{\mathrm{e}}$ et $3^{\text {e }}$ âges. La phéromone soluble dans l'eau est produite au niveau de l'intestin moyen et postérieur et est émise par les fèces et par l'ovipositeur (PROKOPY et al., $1982 b$ ). La conséqu̇ence est le dépôt d'un seul œuf par fruit, une répartition uniforme des œufs dans les arbres (CAMERON \& MORRISSON, 1974) et des vols de dispersion des femelles allant jusqu'à $1000 \mathrm{~m}$ (ROITBERG et al., 1984).

Les réactions d'inhibition de la ponte par la phéromone n'ont pas toujours un caractère absolu. Les jeunes femelles de la mouche des pommes font des erreurs et ont besoin d'apprentissage pour reconnaître un fruit marqué (ROITBERG \& PROKOPY, 1981). Des femelles privées de site de ponte ont aussi tendance à pondre dans des fruits marqués (ROITBERG \& PROKOPY, 1983). La quantité de phéromone déposée peut varier avec les caractéristiques du fruit (taille, espèces...) (PROKOPY, 1972, 1981 $a$ ), phénomène retrouvé chez une autre Tephritidae, Anastrepha fraterculus Wied. (PROKOPY et al., 1982a). D'autres caractères, peut-être génétiques ou nutritionnels, semblent intervenir, car des différences de réaction apparaissent entre populations d'élevage et populations sauvages (PROKOPY, 1981b).

De nombreux autres phytophages marquent aussi l'hôte au moment de la ponte ; PROKOPY ( $1981 a$ et $b$ ) en recense 35 espèces appartenant à 6 ordres. De nouvelles espèces ont été découvertes depuis (MC NEIL \& QUIRRING, 1983). Chez un coléoptère Bruchidae, la détection d'un œuf semble liée aux caractères chimiques et physiques de l'œuf et non à une émission de phéromone après la ponte (MESSINA \& RENWICK, 1985). En Europe, l'existence de phéromones est décrite chez plusieurs ravageurs des plantes: des diptères Tephritidae, la mouche des cerises, $R$. cerasi (HURTER et al., 1976 ; REMUND et al., 1980 ; BOLLER, 1981) et la mouche des fruits, $C$. capitata (PROKOPY et al., 1978), des lépidoptères Pieridae, $P$. brassicae, $P$. rapae (ROTHSCHILD \& SCHOONHOVEN, 1977), chez le coléoptère Curculionidae, Ceuthorrhynchus assimilis Payk. (KOSLOWSKY et al., 1983) et le coléoptère Bruchidae, $A$. obtectus (SzENTESI, 1981). Chez ce dernier, la phéromone serait déposée lors de la ponte sur les grains de haricots stockés, mais pas lors de la ponte dans la nature sur gousses (JARRY \& CHACON, 1983).

Des larves sont aussi capables de signaler leur présence en émettant une phéromone qui éloigne les pon- deuses ; l'exemple le mieux étudié est celui d'Anagasta kuehniella Zell. (Lepid. : Pyralidae) (CORBET, 1971 ; MUDD \& CORBET, 1973).

Les phéromones inhibitrices, associées au dépôt des œufs, sont perçues par olfaction au niveau des antennes et par gustation au niveau des tarses et peut-être de l'ovipositeur chez $P$. brassicae (ROTHSCHILD \& SCHOONHOVEN, 1977 ; BEHAN \& SCHOONHOVEN, 1978 ; DEN OTTER et al., 1980 ; KLIJNSTRA, 1982), par gustation à travers les tarses chez les diptères Tephritidae après l'arrivée sur l'hôte (CRNJAR et al., 1979 ; CRNJAR \& PROKOPY, 1982).

Ces phéromones ont une action intraspécifique, mais celles de certains diptères Tephritidae (PROKOPY et al., 1976 ; PROKOPY, 1981a) et celles des chenilles d'A. kuehniella (MUDD \& CORBET, 1973) ont aussi une activité interspécifique et agissent sur des espèces d'une même famille qui occupent la même niche écologique. La durée d'action de ces phéromones est de quelques jours chez les diptères (PROKOPY, 1981 $a$; RAINA, 1981), et atteint $37 \mathrm{j}$ chez $P$. brassicae (SCHOONHOVEN et al., 1981).

Peu d'investigations ont été réalisées pour isoler et identifier ces phéromones; cependant le travail de MumtaZ \& AliniazeE (1983a et $b$ ) est une tentative dans ce sens.

\section{B. La perception des congénères par des stimulus optiques}

La forme et la couleur des œufs ou des larves renseignent les pondeuses de certains papillons sur la présence de congénères et empêchent la ponte. Les femelles d'Heliconius cydno Doubleday (Lepid. Heliconidae) (WILliaMS \& GILBERT, 1981), de Battus philenor L. (Lepid. Papilionidae) (RAUSHER, 1979), de Pieris sisymbrii Boisduval (Lepid. Pieridae) (SHAPIRO, $1981 a$ et $b$ ) décèlent par la vue les œufs de leur espèce. De façon remarquable, des plantes comme plusieurs espèces de Passifloraceae, hôtes de papillons Heliconidae (GILBERT, 1975 ; WILLIAMS \& GILBERT, 1981) et plusieurs espèces de Cruciferaceae, hôtes de papillons Pieridae (SHAPIRO, $1981 a$ et $b$ ), portent des organes qui imitent les œufs de leurs déprédateurs et qui repoussent les femelles.

Le cas de $P$. brassicae mérite de s'y arrêter (ROTHSCHILD \& SCHOONHOVEN, 1977 ; KLIJNSTRA, 1982) : la présence d'un congénère sur la plante est reconnue à 3 niveaux sensoriels :

1) la vue par la perception de la couleur et de la forme des pontes et/ou par la perception des larves,

2) l'olfaction par la perception de la phéromone pendant une phase de volettement au-dessus d'une feuille,

3) la gustation par la perception de la phéromone par les tarses de la femelle sur la plante.

Chacun de ces niveaux sensoriels peut décider seul - en particulier la vue assure une reconnaissance totale de la présence des œufs - mais leurs actions sont généralement associées.

Ces mécanismes de la reconnaissance spécifique d'un congénère soulignent encore l'importance de la vue dans la découverte d'un site de ponte favorable. 


\section{LE POLYMORPHISME DU COMPORTEMENT DE PONTE}

Un polymorphisme de réactions se manifeste dans le comportement des pondeuses. Il peut dépendre simplement d'un état physiologique ou d'un apprentissage comme nous l'avons vu pour la mouche des pommes. Mais il révèle aussi des variations dans les caractères spécifiques d'un insecte.

Des réactions dissemblables se manifestent dans des populations séparées : en Europe, Pieris rapae émet une phéromone inhibitrice de ponte (ROTHSCHILD \& SCHOONHOVEN, 1977) ; les populations canadiennes et australiennes ne le font pas (IvES, 1978; JONES \& IVES, 1979 ; TRAYNIER, 1979). La mouche de l'oignon est attirée par le di-n-propyl disulfide en Amérique du nord et en Europe, mais pas au Japon (ISHIKAWA et al., 1981). Les populations d'élevage de diptères Tephritidae, C. capitata et $R$. pomonella, ont des comportements, vis-à-vis de la phéromone inhibitrice de ponte, différents de ceux des populations sauvages (PROKOPY, 1981a). Des femelles de C. capitata, originaires de 3 populations ont des préférences différentes dans le choix des fruits (PROKOPY et al., 1984).

Le cas du charançon européen, Rhinocyllus conicus Froel. (Coleopt. Curculionidae), représente un exemple de variations géographiques qui intéresse l'agronome. La femelle pond dans les inflorescences de quelques genres de la famille des composées: Carduus, Cirsium, Silybum. Le choix des pondeuses est spécialisé selon les régions. En France, il se porte sur Cirsium vulgare (Savi) Ten. (= C. lanceolatum (L.) Scop.) dans le sud-ouest, sur Carduus nutans L. en Alsace, en Bourgogne, dans le Jura ; $C$. vulgare et C. nutans sont tous les 2 colonisés dans le Berry. La population du charançon des environs d'Arles est monophage sur Silybum marianum Gaertn. etc... Cette diversité dans le choix du végétal-hôte serait sous contrôle génétique. Rh. conicus est utilisé en lutte biologique pour limiter différents chardons européens introduits en Amérique du nord. L'expérience montre que, pour avoir une action efficace, il faut importer l'écotype du charançon adapté à l'espèce de chardon à détruire (ZWÖLFER \& PREISS, 1983 ; ZWÖLFER \& HARRIS, 1984).

Un polymorphisme se manifeste aussi entre individus d'une même population. Chez certaines espèces, la variabilité apparaît en absence de plantes-hôtes. Les femelles de la bruche du haricot (LABEYRIE, 1961) et celles de la teigne de la betterave (ROBERT, 1965, 1970) pondent toutes en présence de leur hôte; en absence de ce dernier, seule une partie des insectes émet normalement des œufs. Chez la teigne de la betterave, la proportion des pondeuses affranchies de la plante nourricière varie sensiblement d'une année à l'autre, de 20 à 70 p. 100 (ROBERT et al., 1977).

Chez d'autres espèces le polymorphisme est révélé par les réactions des pondeuses vis-à-vis des différentes espèces de plantes-hôtes dans un écosystème. Les stimulus des différents végétaux supports de ponte n'ont pas la même valeur pour toutes les femelles. Papilio machaon pond sur diverses ombellifères ; certaines femelles déposent leurs œufs sur toutes les ombellifères-hôtes en les choisissant selon une hiérarchie bien établie ; elles ont un comportement d'oligo- phages. D'autres individus ont une stratégie de monophages et ne pondent que sur une seule espèce d'ombellifères (WIKLUND, 1981). Des phénomènes analogues sont décrits chez d'autres lépidoptères, des Pieridae : Colias meadii Edw. (STANTON, 1979), C. philodice eriphyle Edw. (STANTON \& COOK, 1984), C. eurytheme Bdv. (TABASHNIK et al., 1981), un Nymphalidae : Euphydrias editha (SINGER, 1983).

Quels mécanismes sont responsables de ces variations dans le choix des femelles? On peut avec TABASHNIK et al. (1981) distinguer 3 hypothèses : 1) conditionnement des larves, 2) conditionnement des adultes, 3) patrimoine génétique.

\section{Conditionnement larvaire (ou «Hopkins host selection principle »)}

Les femelles choisiraient préférentiellement les plantes sur lesquelles elles se sont alimentées à l'état larvaire. Aucune recherche expérimentale n'apporte de preuve à ce principe (WIKLUND, 1974 ; JONES \& IVES, 1979 ; STANTON, 1979 ; TABASHNIK et al., 1981 ; JAENIKE, 1982 ; PROKOPY et al., 1982c ; BOWERS, 1983 ; SingER, 1983 ; WILliams, 1983). Des erreurs d'interprétation peuvent faire croire au conditionnement larvaire si l'on ne tient pas compte de tous les composants du comportement de ponte (WASSERMAN, 1981).

\section{Conditionnement des femelles}

La préférence de la femelle est influencée par ses contacts avec la $1^{\text {re }}$ espèce-hôte rencontrée à l'état adulte. Un tel apprentissage est bien mis en évidence chez un phytophage, $R$. pomonella, (PROKOPY et al., $1982 c)$ : des femelles, développées sur pomme, pondent de préférence sur fruit de Crataegus mollis Scheele ; mais si les $1^{\text {res }}$ expériences de ponte se réalisent sur pomme, le choix se portera ensuite sur pomme et le Crataegus sera délaissé. JAENIKE (1982), expérimentant sur 4 espèces de drosophiles, montre qu'au moment de la ponte le choix du support de ponte est modifié par l'expérience des femelles et non par celle des larves.

\section{Patrimoine génétique}

Les variations de la ponte ont une base génétique chez la bruche du haricot (LABEYRIE, 1961). La hiérarchie préférentielle des hôtes de Colias eurytheme serait également génétique et contrôlée par plusieurs gènes (TABASHNIK et al., 1981). D'autres recherches confortent cette idée (Hovanitz, 1969 ; PHILIPS \& BARNES, 1975 ; SINGER, 1983 ; ZWÖLFER \& PREISS, 1983 ; PROKOPY et al., 1982c, 1984).

Les différences individuelles de réaction aux stimulus émis par les diverses plantes-hôtes ont des conséquences importantes en écologie et en agronomie. Les populations ont la capacité de coloniser des hôtes nouveaux, de se maintenir quand les meilleurs hôtes sont rares ou absents. Le carpocapse des prunes est capable de pondre sur des hôtes inhabituels, cerises ou pommes, lorsque les prunes sont rares (BOVEY, 1966b). Au cours de ce siècle, le carpocapse des pommes a colonisé les vergers de noix, d'abricots et de prunes (BOVEY, 1966a; PHILIPS \& BARNES, 1975). 
On peut penser que des individus dans une population auront l'aptitude d'engendrer des écotypes capables de contourner certains types de résistance chez les végétaux. Il est encore trop tôt pour établir des lois générales, mais le polymorphisme de réactions des pondeuses doit être retenu dans toutes les études d'éthologie, d'écologie et d'agronomie.

\section{LA STIMULATION DE L'OVOGENÈSE PAR LA PLANTE-HÔTE}

Les recherches sur les liens chimiques entre femelles pondeuses et plantes-hôtes ont entraîné la découverte d'un phénomène non soupçonné : la stimulation de l'ovogenèse en présence de plante-hôte par gustation et olfaction sans qu'il y ait prise alimentaire. Ainsi l'activité ovarienne est maximale lorsqu'un site végétal favorable à la ponte est présent. Un petit nombre de cas sont connus (annexe 3 ) mais ce type de relations plante-insecte est certainement plus répandu.

\section{LES APPLICATIONS EN AGRICULTURE}

L'emploi des kairomones et des allomones agissant sur les différentes étapes de la ponte est envisagé pour entraver la reproduction et les activités des insectes phytophages ravageurs. Les possibilités d'utilisation ont été discutées par POUZAT (1980) et JACKSON \& LEWIS (1981).

Les kairomones guident les femelles vers la plante support de ponte ; cette action est comparable à celle des phéromones qui conduisent un insecte vers son partenaire sexuel. Leur emploi est expérimenté pour attirer les femelles dans des pièges comportant un diffuseur de composés volatils associés à un bâti de forme et de couleur adaptées à chaque espèce. Quelques réalisations peuvent être signalées pour la mouche du chou (FINCH et al., 1980 ; FINCH \& SKINNER, $1982 a$; DAPSIS \& FERRO, 1983), la mouche de la carotte (GUERIN \& STÄDLER, 1982), la mouche des pommes (REISSIG et al., 1982, 1985). Leur efficacité permet de les utiliser pour la prévision de dégâts. La connaissance du comportement d'émission des œufs peut aussi entraîner la création de pièges à œufs comme FREULER \& FISCHER (1983) le proposent pour la mouche du chou.

La ponte est une phase vulnérable dans le cycle d'un insecte. A l'intérieur d'une espèce végétale, il existe des variétés qui n'attirent pas ou peu les pondeuses ; elles sont protégées par des allomones et (ou) par des caractères physiques. Les programmes de sélection des plantes doivent incorporer ces résistances chimiques (POUZAT, 1980 ; BECK \& SCHOONHOVEN, 1980 ; RENWICK, 1983 ; JACKSON et al., 1984) ou optiques (PROKOPY \& OWENS, 1983 ; PROKOPY et al., $1983 a$ et $b$ ) afin de limiter la colonisation par les femelles.

La recherche de composés végétaux inhibiteurs de ponte est aussi entreprise pour perturber les pondeuses et les éloigner par traitement des cultures. Quelques essais montrent des possibilités d'utilisation (FLINT et al., 1977, 1978 ; ROBERT \& BLAISINGER, 1978). La mise en évidence de substances volatiles végétales acti- ves (SAXENA \& BASIT, 1982a et $b$ ) offre un champ expérimental nouveau.

Les cultures en mélange de 2 ou plusieurs espèces cultivées, possibles sur de petites parcelles, posent des problèmes en agriculture intensive mécanisée (RISH et al., 1983 ; CAPINERA et al., 1985).

Il est tentant d'appliquer dans les vergers ou dans les cultures les phéromones inhibitrices de la ponte. Des essais réalisés en Suisse contre la mouche des cerises, $R$. cerasi, donnent des espoirs (KaTsOYANNOS \& BOLLER, 1976, 1980 ; BOLLER, 1981). Leur utilisation semble limitée à des cas particuliers (BOLLER, 1981 ; PROKOPY, $1981 a$ et $b$ ).

L'intégration des sémiochimiques et des stimulus physiques des végétaux dans la prévention contre les insectes ravageurs dépend d'une meilleure compréhension de leur rôle dans les interactions établies dans les écosystèmes. Ce sont des recherches longues à coût élevé qui sont trop peu développées aujourd'hui.

\section{CONCLUSIONS}

L'appel de KoGAN au $15^{\text {e }}$ congrès international d'entomologie à Washington en 1976 a été entendu ; les recherches sur la ponte des insectes phytophages se sont multipliées et les 3/4 des travaux analysés dans cette revue sont postérieurs à 1975. Beaucoup de résultats apportent des données inédites en physiologie, éthologie, écologie et en agronomie.

Le rôle primordial des kairomones dans la reconnaissance des plantes-hôtes par les femelles gravides est confirmé, mais cette reconnaissance n'est pas une réponse à des stimulus chimiques simples, elle intègre une constellation de stimulus chimiques spécifiques, olfactifs et gustatifs, avec des manifestations de synergie. Des substances comme l'eau et des nutriments pourraient aussi y coopérer. Il est maintenant incontestable que des allomones des plantes-non-hôtes, mais aussi des allomones de plantes-hôtes, jouent un rôle d'inhibition de la ponte et assurent la dispersion des femelles gravides. A partir de ces données, on peut essayer d'établir des définitions des plantes-hôtes et des plantes-non-hôtes.

Une plante-hôte est caractérisée par la présence de kairomones spécifiques, par l'absence d'allomones répulsives et inhibitrices. Des allomones peuvent préexister dans l'hôte, mais elles ne sont pas directement perceptibles par l'insecte ; elles sont révélées par l'action de congénères après lésion du végétal.

Trois types de plantes-non-hôtes peuvent être décrits :

1) des végétaux pourvus d'attractifs et de stimulants combinés avec des répulsifs et des inhibiteurs (ROTHSCHILD \& SCHOONHOVEN, 1977 ; CHEW, 1977a) ;

2) la grande masse des végétaux non-hôtes, dépourvus d'attractifs et de stimulants et équipés de répulsifs et d'inhibiteurs ;

3) des plantes qui manquent à la fois d'attractifs et de stimulants, de répulsifs et d'inhibiteurs. De telles plantes semblent rares. ICHINOSE \& SASAKI (1975), JERMY \& SZENTESI (1978) en citent des exemples.

Cette classification reflète les connaissances actuelles, mais elle est certainement simpliste quand on songe à la masse des informations sensorielles qu'une 
femelle intègre pour mener avec succès les différentes étapes de la ponte, car les stimulus physiques sont associés intimement aux stimulus chimiques et ont un rôle plus important qu'il n'était imaginé. Il faut faire une part particulière aux stimulus optiques qui agissent à toutes les étapes de la ponte, dans l'attraction lointaine, l'atterrissage et l'émission des œufs. Des résultats récents révèlent des aspects originaux de leur action. Des stimulus visuels ont un effet de synergie sur l'activité de stimulus chimiques. La vue peut être seule impliquée dans l'identification d'un congénère occupant déjà un site de ponte et, sans doute aussi, dans la reconnaissance de l'espèce végétale, voire d'individus particuliers à l'intérieur d'une espèce végétale. Il paraît difficile de dissocier les messages chimiques des messages physiques.

D'autres résultats apportent des connaissances inédites :

- la stimulation de l'activité ovarienne par les kairomones de l'hôte,
- les mécanismes fascinants mis en œuvre pour empêcher les pontes multiples sur un hôte et éviter la compétition en manipulant des allomones trouvées dans l'hôte ou en utilisant des phéromones,

- des données sur le polymorphisme du comportement de ponte manifesté, d'une part, entre populations et, d'autre part, entre individus d'une population. Cette variabilité pose immédiatement des interrogations de physiologie sensorielle et aussi de génétique et doit être prise en compte par les agronomes.

Dans tous ces domaines des relations plantespondeuses, les résultats laissent encore beaucoup d'inconnues. Il faut poursuivre les recherches qui apporteront de nouvelles découvertes, sources de solutions aux problèmes des ravageurs en agriculture.

Recu le 12 mars 1985 Accepté le 17 septembre 1985.

\section{ANNEXE 1}

Insectes dont la ponte est stimulée par des kairomones de leurs hôtes (non cités dans le texte).

\section{APPENDIX 1}

Insects with egg-laying stimulated by host kairomones (not mentioned in the text)

Lepidoptera

Papilionidae

Papilio protenor demetrius Cram. Ichinose \& Honda, 1978

$P$. demeolus $\mathrm{L}$. Saxena \& Goyal, 1978

P. bianor dehaanii C. \& R. Felder Abe et al., 1981

P. xuthus L. Abe et al., 1981

Sphingidae

Protoparce sexta Johan

Tortricidae

Adoxophyes orana F. R.

Laspeyresia pomonella $\mathrm{L}$.

Zeiraphera diniana Guén. Grapholitha funebrana Tr. Keiferia lycopersicella Walsh.

Yamamoto \& Fraenkel, 1960

Yamamoto et al., 1969

Sparks, 1973

Den Otter et al., 1978

Sutherland et al., 1977

Benz, 1969

Deseö, 1967

Burton \& Schuster, 1981

Choristoneura fumiferana Clém. Städler, 1974

Pyralidae

Amyelois transitella Walker

Ephestia cautella Walker

Diaphana nitidalis Stoll.

Curtis \& Clark, 1979

Barrer \& Jay, 1980

Elsey \& Mc Fadden, 1981
Noctuidae

Anadevidia peponis Fabr. Ichinose \& Sasaki, 1975 Earias fabia Stoll.

Mehta \& Saxena, 1970

Diptera

Tephritidae

Dacus tryoni Froggat.

Philophylla heraclei $\mathrm{L}$

Anastrepha suspensa Loew.

Chloropidae

Oscinella frit $\mathrm{L}$.

Leroi, 1975

Szentesi et al., 1979

Hamilton et al., 1979

Anthomyiidae

Hylemyia cilicrura Rond. Barlow, 1965

Pegomya betae Curt.

Röttger, 1979a

Coleoptera

Curculionidae

Ceuthorrhynchus maculaalba Herbst. Saringer, 1976

\section{ANNEXE 2}

Insectes sensibles à des allomones inhibitrices de ponte (non cités dans le texte).

APPENDIX 2

Insects sensitive to oviposition-inhibiting allomones (not mentioned in the text).

\section{Lepidoptera}

Noctuidae

Heliothis virescens $\mathrm{F}$.

Spodoptera litura $\mathrm{F}$.

Spodoptera frugiperda J.E. Smith

S. eridiana Cramer

S. ornithogalli Guén.

Pyralidae

Crocidolimia binotalis Zell.

Gelechiidae

Pectinophora gossypiella Saunders

Phthorimaea operculella Zell.
Tingle \& Mitchell, 1984

Joshi \& Sitaramaiah, 1979

Burnett et al., 1978

Falgoonee, 1981

Flint et al., 1977

Sharma et al., 1981
Diptera

Hylemyiidae

Hylemya antiqua Meig. Wiens et al., 1978

Tephritidae

Rhagoletis cerasi $\mathrm{L}$.

Anastrepha suspensa Loew.

Dacus oleae Gmel.

Dacus dorsalis Hendel

Ceratitis capitata Wiedemann

Dacus cucurbitae Coquillet
Alfaro et $a^{\prime},, 1981$

Haisch \& Levinson, 1980

Szentesi et al., 1979

Prokopy \& Hanniotakis, 1976

Seo et al., 1983 


\section{ANNEXE 3}

Insectes phytophages dont l'ovogenèse est stimulée par des kairomones de plantes support de ponte. APPENDIX 3

Phytophagous insects with ovogenesis stimulated by kairomones formed by their host-plants.

plantes-hôtes

Lepidoptera

Plutellidae

Acrolepiopsis assectella Zell.

Plutella maculipennis Curt.

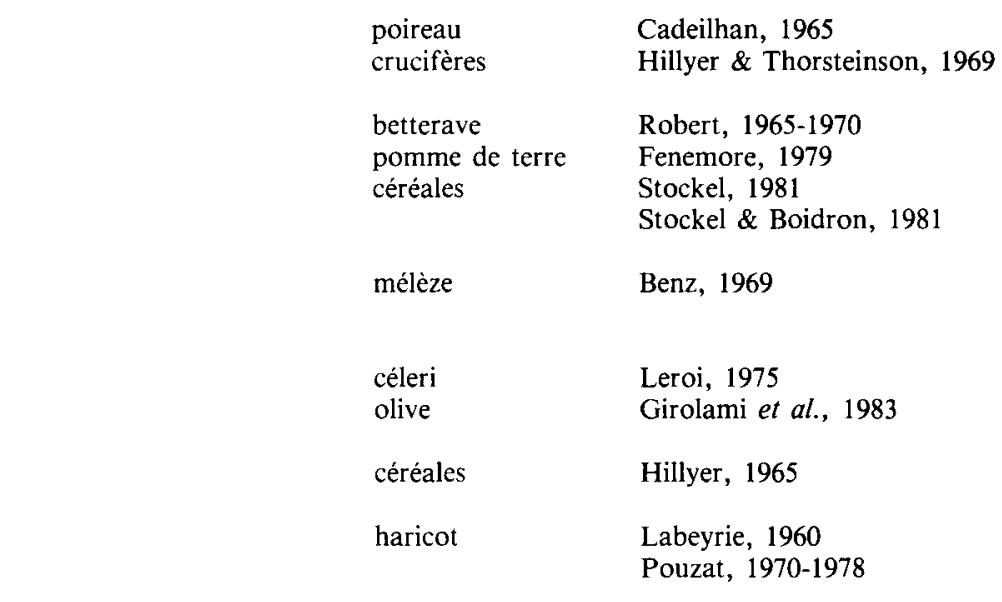

Gelechiidae

Scrobipalpa ocellatella Boyd.

Phthorimaea operculella Zell.

Sitotroga cerealella Oliv.

Pouzat, $1970-1978$

Tortricidae

Zeiraphera diniana Guén.

Diptera

Tephritidae

Philophylla heraclei L.

Dacus oleae Gmel

Chloropidae

Oscinella frit $\mathrm{L}$.

Coleoptera Bruchidae

Acanthoscelides obtectus Say.

\section{RÉFÉRENCES BIBLIOGRAPHIQUES}

Abe H., Teramoto Y., Ichinose T., 1981. Relationship between host plant ranges of the three papilionid butterflies and ovipositioninducing contact chemicals in their host plants. Appl. Entomol. Zool., 16, 493-496.

Ahman I., 1984. Oviposition and larval performance of Rabdophaga terminalis on Salix spp. with special consideration to bud size of host plants. Entomol. exp. appl., 35, 129-136.

Alfaro R. I., Pierce H. D., Borden J. H., Oehlschlaeger A. C., 1981. Insect feeding and oviposition deterrents from western red cedar foliage. $J$. chem. Ecol., 7, 39-48.

Auger J., Thibout E. T., 1981. Influences comparées du contact avec les substances volatiles spécifiques d'Allium porrum et avec leurs précurseurs sur la ponte de la teigne du poireau Acrolepiopsis assectella Zell. (Lepidoptera, Hyponomeutö̈dae). Acta Oecol., Oecol. appl., 2, 267-276.

Auger J., Thibout E., 1983. Spécificité des substances non volatiles des Allium responsables de la ponte de la teigne du poireau Acrolepiopsis assectella (Lepidoptera). Entomol. exp. appl., 34, 71-77.

Barlow C. A., 1965. Stimulation of oviposition in the seed-corn maggot fly, Hylemya cilicrura (Rond) (Diptera : Anthomyiidae). Entomol. exp. appl., 8, 83-95.

Barrer P. M., Jay E. G., 1980. Laboratory observations on the ability of Ephestia cautella (Walker) (Lepidoptera : Phycitidae) to locate, and to oviposit in response to a source of grain odour. $J$. stored Prod. Res., 16, 1-7.

Beck S. D., 1965. Resistance of plants to insects. Annu. Rev. Entomol., 10, 207-232.

Beck S. D., Schoonhoven L. M., 1980. Insect behavior and plant resistance, 115-136. In F. G. Maxwell, P. P. Jennings : « Breeding plants resistant to insects », J. Wiley and sons, New York, $700 \mathrm{p}$.

Behan M., Schoonhoven L. M., 1978. Chemoreception of an oviposition deterrent associated with eggs in Pieris brassicae. Entomol. exp. appl., 24, 163-179.

Benz G., 1969. Influence of mating, insemination and other factors on oogenesis and oviposition in the moth Zeiraphera diniana. $J$. Insect Physiol., 15, 55-71.

Boller E. F., 1981. Oviposition-deterring pheromone of the European cherry fruit fly. Status of research and potential applications, 457-462. In E. R. Mitchell : « Management of insect pests with semio-chemicals: concept and practice", Plenum Press, New York, $514 \mathrm{p}$.
Boller E. F., Prokopy R. J., 1976. Bionomics and management of Rhagoletis. Annu. Rev. Entomol., 21, 223-246.

Bovey P., 1966a. Le carpocapse ou le ver des pommes et des poires, 653-734. In A. S. Balachowsky : "Entomologie appliquée à l'agriculture, Lépidoptères ", Tome 2, Masson Ed. Paris, 1057 p.

Bovey P., 1966b. Le carpocapse des prunes, 746-763. In A. S. Balachowsky : “Entomologie appliquée à l'agriculture, Lépidoptères », Tome 2, 746-763, Masson Ed. Paris, 1057 p.

Bowers M. D., 1983. The role of iridoid glycosides in host-plant specificity of checkerspot butterflies. J. chem. Ecol., 9, 475-493.

Brunel E., 1977. Etude de l'attraction périodique de femelles de Psila rosae Fabr. par la plante-hôte et influence de la végétation environnante. Coll. int. C.N.R.S., $n^{\circ}$ 265, 373-389 "Comportement des insectes et milieu trophique », $493 \mathrm{p}$.

Burnett B. C., Jones S. B., Mabry T. J., 1978. The role of sesquiterpene lactones in plant-animal coevolution, 233-257. In : J. B. Harborne : « Biochemical aspects of plant-animal coevolution 》, Academic Press, New York, 435 p.

Burton R. L., Schuster D. J., 1981. Oviposition stimulant for tomato pinworms from surfaces of tomato plants. Ann. Entomol. Soc. Am., 74, 512-515.

Cadeilhan L., 1965. Action de la plante-hôte sur l'ovogenèse de l'adulte d'Acrolepia assectella Zell. (Insecte Lépidoptère). C. $R$. Acad. Sci., Paris, Sér. D, 261, 1910-1913.

Cameron P. J., Morrison F. O., 1974. Sampling methods for estimating the abundance and distribution of all life stages of the apple maggot Rhagoletis pomonella. Can. Entomol., 106, 1025-1034.

Capinera J. L., Weissling T. J., Schweizer E. E., 1985. Compatibility of intercropping with mechanized agriculture : effects of strip intercropping of pinto beans and sweet corn on insect abundance in Colorado. J. econ. Entomol., 78, 354-357.

Chapman R. F., 1974. The chemical inhibition of feeding by phytophagous insects : a review. Bull. entomol. Res., 64, 339-363.

Chew F. S., 1977a. Coevolution of Pierid butterflies and their cruciferous foodplants. II. The distribution of eggs on potential foodplants. Evolution, 31, 568-579.

Chew F. S., 1977b. The effects of introduced mustard (Cruciferae) on some native North American cabbage butterflies (Lepidoptera : Pieridae). Atala, 5, 13-19.

Cirio V., 1971. Reperti sul meccanismo stimulo-riposta nell' ovide- 
posizione del Dacus oleae Gmelin (Diptera : Trypetidae). Redia, 52, 577-600.

Claridge M. F., Wilson M. R., 1978. Oviposition behaviour as an ecological factor in woodland canopy leaf hoppers. Entomol. exp. appl., 24, 301-309.

Coaker T. H., 1980. Insect pest management in Brassica crops by inter-cropping. Bull. SROP, 3, 117-125.

Cobbinah J. R., Morgan F. D., Douglas T. J., 1982. Feeding responses of the gum leaf skeletoniser Uraba lugens Walker to sugars, aminoacids, lipids, sterols, salts, vitamins and certain extracts of eucalypt leaves. J. Aust. entomol. Soc., 225-236.

Corbet S. A., 1971. Mandibular gland secretion of larvae of the flour moth Anagasta kuehniella contains an epideictic pheromone and elicits oviposition movements in a hymenopteran parasite. Nature, 232, 481-484.

Courtney S. P., 1982. Coevolution of pierid butterflies and their cruciferous foodplants. IV. Crucifer apparency and Anthocharis cardamines L. oviposition. Oecologia, 52, 258-265.

Crnjar R. M., Prokopy R. J., 1982. Morphological and electrophysiological mapping of tarsal chemoreceptors of ovipositiondeterring pheromone in Rhagoletis pomonella flies. J. Insect Physiol., 28, 393-400.

Crnjar R. M., Prokopy J., Dethier V. G., 1979. Electrophysiological identification of oviposition-deterring pheromone receptors in Rhagoletis pomonella (Diptera: Tephritidae). J. New York Entomol. Soc., 86, 283-284.

Curtis C. E., Clark J. D., 1979. Responses of navel orangeworm moths to attractants evaluated as oviposition stimulants in an almond orchard. Environ. Entomol., 8, 330-333.

Dapsis L. J., Ferro D. N., 1983. Effectiveness of baited cone traps and colored sticky traps for monitoring adult cabbage maggots, with notes on female ovarian development. Entomol. exp. appl., 33, $35-42$

Derridj S., Fiala V., 1983. Sucres solubles des feuilles de maïs (Zea mays L.) et oviposition de la pyrale (Ostrinia nubilalis $\mathrm{Hbn}$.). C. $R$. Acad. Agric. Fr., 69, 465-472.

Deseö K. V., 1967. The role of olfactorial stimuli in the egg-laying behaviour of plum moth (Grapholitha funebrana Tr.). Acta phytopathol. Acad. Sci. Hung., 2, 243-250.

Dethier V. G., 1947. Chemical insect attractants and repellents. Blakiston Co, Philadelphia, 289 p.

Dindonis L. L., Miller J. R., 1980a. Host-finding behavior of onion flies, Hylemya antiqua. Environ. Entomol., 9, 769-772.

Dindonis L. L., Miller J. R., $1980 b$. Host-finding response of onion and seedcorn flies to healthy and decomposing onions and several synthetic constituents of onion. Environ. Entomol., 9, 467-472.

Dittrick L. E., Jones R. L., Chiang H. C., 1983. An oviposition deterrent for the European corn borer Ostrinia nubilalis (Lepidoptera : Pyralidae) extracted from larval frass. J. Insect. Physiol., 29, 119-121.

Ellis P. R., Hardman J. A., Crisp P., Johnson A. G., 1979. The influence of plant age on resistance of radish to cabbage root fly egg-laying. Ann. appl. Biol., 93 (2), 125-131.

Ellis P. R., Taylor J. D., Littlejohn I. H., 1982. The role of microorganisms colonising radish seedlings in the oviposition behaviour of cabbage root fly, Delia radicum. Proc. 5th int. Symp. Insect-Plant Relationships, pp. 131-137, Visser J. H., Minks A. K. ed., Pudoc Wageningen, 464 p.

Elsey K. D., McFadden T. L., 1981. Pickleworm : oviposition on an artificial surface treated with curcubit extract. $J$. econ. Entomol., 74, 473-474.

Falgoonee I., 1981. Behavioral response of Crocidolomia binotalis to neem. Proc. Ist Int. Neem Conf., 109-120, H. Schmutterer, K. R. S. Ascher, H. Rembold ed., G.T.Z. publ., 6236 Eschborn 1, Germany, 297 p.

Feeny P., 1975. Biochemical coevolution between plants and their insect herbivores, 3-19. In L. E. Gilbert \& R. H. Raven : " Coevolution of animals and plants », University of Texas Press, $246 \mathrm{p}$.

Fenemore P. G., 1979. Oviposition of potato tuber moth, Phthorimaea operculella Zell. (Lepidoptera : Gelechiidae) ; the influence of adult food, pupal weight, and host-plant tissue on fecundity. $N$. $Z$. J. Zool., 6, 389-395.
Fenemore P. G., 1980. Oviposition of potato tuber moth, Phthorimaea operculella Zell. (Lepidoptera : Gelechiidae); identification of host-plant factors influencing oviposition response. $N . Z . J$. Zool., 7, 435-439.

Feron M., 1962. L'instinct de reproduction chez la mouche méditerranéenne des fruits, Ceratitis capitata Wied. (Dipt. Trypetidae) : comportement sexuel, comportement de ponte. Rev. Pathol. veg. Entomol. agric. Fr., 41, 1-129.

Finch S., 1977. Effect of secondary plant substances on host-plant selection by the cabbage root fly. Coll. int. C.N.R.S., $n^{\circ} 265,251$ 267, Comportement des insectes et milieu trophique, $493 \mathrm{p}$.

Finch S., 1978. Volatile plant chemicals and their effect on host plant finding by the cabbage root fly (Delia brassicae). Entomol. exp. appl., 24, 350-359.

Finch S., 1980. Chemical attraction of plant-feeding insects to plants. Appl. Biol., 5, 67-143.

Finch S., Skinner G., 1982a. Trapping cabbage root flies in traps baited with plant extracts and with natural and synthetic isothiocyanates. Entomol. exp. appl., 31, 133-139.

Finch S., Skinner G., 1982b. Upwind flight by the cabbage root fly, Delia radicum. Physiol. Entomol., 7, 387-399.

Finch S., Freuler J., Städler E., 1980. Trapping Hylemya brassicae adults. Bull. SROP, 3, 11-17.

Fitt G. P., 1984. Oviposition behaviour of two tephritid fruit flies, Dacus tryoni and Dacus jarvisi, as influenced by the presence of larvae in the host fruit. Oecologia, 62, 37-46.

Fletcher B. S., Watson C. A., 1974. The ovipositional response of the tephritid fruit fly, Dacus tryoni, to 2-chloro-ethanol in laboratory bioassays. Ann. Entomol. Soc. Am., 67, 21-23.

Flint H. M., Smith R. L., Pomonis J. G., Forey D. E., Horn B. R., 1977. Phenylacetaldehyde : oviposition inhibitor for the pink bollworm. J. econ. Entomol., 70, 547-548.

Flint H. M., Noble J. M., Shaw D., 1978. Phenylacetaldehyde : tests for control of the pink bollworm and observations on other Lepidoptera infesting cotton. J. Georgia Entomol. Soc., 13, 284 289.

Fraenkel G. S., 1959. The raison d'être of secondary plant substances. Science, 129, 1466-1470.

Fraenkel G. S., 1969. Evaluation of our thoughts on secondary plant substances. Entomol, exp. appl., 12, 473-486.

Freuler J., Fischer S., 1983. Le piège à œuf, nouveau moyen de prévision d'attaque pour la mouche du chou, Delia radicum brassicae (L.). Rev. Suisse vitic. arboric. hortic., 15, 107-110.

Gilbert L. E., 1975. Ecological consequences of a coevoluted mutualism between butterflies and plants, 210-240. In L. E. Gilbert \& P. H. Raven : " Coevolution of animals and plants », University of Texas Press, 246 p.

Girolami V., Vianello A., Strapazzon A., Ragazzi E., Veronese G., 1981. Ovipositional deterrents in Dacus oleae. Entomol. exp. appl., 29, $177-188$.

Girolami V., Strapazzon A., de Gerloni P. F., 1983. Insect/plant relationships in olive flies : general aspects and new findings, 258267. In R. Cavalloro: "Fruit flies of economic importance », Proc. CEC/IOBC intern. Sympos., Athens, Nov. 1982, A. A. Balkena, Rotterdam, $642 \mathrm{p}$.

Grove J. F., Blight M. M., 1983. The oviposition attractant for the mushroom phorid Megaselia halterata : the identification of volatiles present in mushroom house air. J. Sci. Food Agric., 34, 181185.

Guérin P., 1980. La mouche de la carotte. Les substances volatiles de la plante-hôte comme moyens de contrôle. In : "Les phéromones sexuelles et les médiateurs chimiques chez les insectes : utilisation en lutte intégrée ", p. 197, Les Colloques de I'INRA, 3, Colmar, 25-27 nov. 1980, 206 p.

Guérin P., Städler E., 1982. Host odour perception in three phytophagous Diptera. A comparative study. Proc. Sth int. Symp. InsectPlant Relationships, 95-105, Visser J. H., Minks A. K. ed., Pudoc Wageningen, $464 \mathrm{p}$.

Guérin P., Städler E., Buser H. R., 1983. Identification of host plant attractants for the carrot fly, Psila rosae. J. chem. Ecol., 9, 843-861.

Gupta P. D., Thorsteinson A. J., 1960. Food plant relationships of the diamond-back moth (Plutella maculipennis (Curt.)). II. Sensory 
regulation of oviposition of the adult female. Entomol. exp. appl., 3, 305-314.

Haisch A., Levinson H. Z., 1980. Influences of fruit volatiles and coloration on oviposition of the cherry fruit fly. Naturwissenschaften, 67, 44-45.

Hamilton R. J., Munro J., Rowe J. M., 1979. The identification of chemicals involved in the interaction of Oscinella frit with Avena sativa. Entomol. exp. appl., 25, 328-341.

Haniotakis G. E., Voyadjoglou A., 1978. Oviposition regulation in Dacus oleae by various olive fruit characters. Entomol. exp. appl., 24, 387-392.

Hardman J. A., Ellis P. R., 1978. Host plant factors influencing the susceptibility of cruciferous crops to cabbage root fly attack. Entomol. exp. appl., 24, 393-397.

Harris M. O., Miller J. R., 1982. Synergism of visual and chemical stimuli in the oviposition behaviour of Delia antiqua. Proc. 5th int. Symp. Insect-Plant Relationship, 117-122, Visser J. H., Minks A. K. ed., Pudoc Wageningen, 464 p.

Harris M. O., Miller J. R., 1983. Color stimuli and oviposition of the onion fly, Delia antiqua (Meigen) (Diptera : Anthomyiidae). Ann. Entomol. Soc. Am., 76, 766-771.

Hawkes C., Coaker T. H., 1979. Factors affecting the behavioural responses of the adult cabbage root fly, Delia brassicae to host plant odour. Entomol. exp. appl., 25, 45-48.

Hillyer R. J., 1965. Individual variation in ovary development and in reproductive behaviour of Oscinella frit. Proc. XII Int. Congr. Entomol. London, p. 390.

Hillyer R. J., Thorsteinson A. J., 1969. The influence of the host plant or males on ovarian development or oviposition in the diamondback moth Plutella maculipennis (Curt.). Can. J. Zool., 47, 805-816.

Hough J. A., Harman G. E., Eckenrode C. J., 1981. Microbial stimulation of onion maggot oviposition. Environ. Entomol., 10, 206210.

Hovanitz W., 1969. Inherited and/or conditioned changes in hostplant preference in Pieris. Entomol. exp. appl., 12, 729-735.

Hurter J., Katsoyannos B., Boller E. F., Wirz P., 1976. Beitrag zur Anreicherung und teilweisen Reinigung des eiablageverhindernden Pheromons der Kirschenfliege, Rhagoletis cerasi L. Z. angew. Entomol., 80, 50-56.

Ichinose T., Honda H., 1978. Ovipositional behavior of Papilio protenor demetrius Cramer and the factors involved in its host plants. Appl. Entomol. Zool., 13, 103-114.

Ichinose T., Sasaki M., 1975. An experimental analysis and integrated evaluation of various factors involved in the host plant specificity of the cucumber looper, Anadevidia peponis (Fabricius) (Lepidoptera : Noctuidae). Appl. Entomol. Zool., 10, 284-297.

Ilse D., 1937. New observations on responses to colours in egglaying butterflies. Nature, 140, 544-545.

Ishikawa Y., Ikeshoji T., Matsumoto Y., 1978. A propylthio moiety essential to the oviposition attractant and stimulant of the onion fly, Hylemyu antiqua Meigen. Appl. Entomol. Zool., 13, 115-122.

Ishikawa Y., Ikeshoji T., M.tsumoto Y., Tsutsumi M., Mitsui Y., 1981. Field trapping of the $\mathrm{Oi}^{-}$ion and seed-corn flies with baits of fresh and aged onion pulp. Appl. Entomol. Zool., 16, 490-493.

Ives P. M., 1978. How discriminating are cabbage butterflies. Aust. J. Ecol., 3, 261-276.

Jackai L. E. \& Singh, 1981. Studies on some behavioral aspects of Maruca testulalis on selected species of Crotalaria and Vigna unguiculata. Trop. Grain Legume Bull., 22, 3-6.

Jackson R. D., Lewis W. J., 1981. Summary of significance and employment strategies for semiochemicals, 283-295. In D. A. Nordlund, R. L. Jones, W. J. Lewis : « Semiochemicals : their role in Pest control ", Wiley New York, 306 p.

Jackson D. M., Severson R. F., Johnson A. W., Chaplin J. F., Stephenson M. G., 1984. Ovipositional response of tobacco budworm moths (Lepidoptera: Noctuidae) to cuticular chemical isolates from green tobacco leaves. Environ. Entomol., 13, 1023-1030.

Jaenike J., 1982. Environmental modification of oviposition behavior in Drosophila. Am. Nat., 119, 784-802.

Jarry M., Chacon A., 1983. Influence de la durée d'exposition des gousses mûres de Phaseolus vulgaris sur la distribution des pontes
d'Acanthoscelides obtectus en plein champ. Entomol. exp. appl., 33, 213-219.

Jermy T., Szentesi A., 1978. The role of inhibitory stimuli in the choice of oviposition site by phytophagous insects. Entomol. exp. appl., 24, 458-471.

Jones R. E., Ives P. M., 1979. The adaptiveness of searching and host selection behaviour in Pieris rapae. Aust. J. Ecol., 4, 75-86.

Joshi B. G., Sitaramaiah S., 1979. Neem kernel as an ovipositional repellent for Spodoptera litura (F.) moths. Phytoparasitica, 7, 199202.

Kamm J. A., Butery R. G., 1983. Response of the alfalfa seed chalcid, Bruchophagus roddi, to alfalfa volatiles. Entomol. exp. appl., 33, 129-134.

Katsoyannos B. I., Boller E. F., 1976. First field application of oviposition-deterring marking pheromone of European cherry fruit fly. Environ. Entomol., 5, 151-152.

Katsoyannos B. I., Boller E. F., 1980. Second field application of oviposition-deterring pheromone of the European cherry fruit fly, Rhagoletis cerasi L. (Diptera : Tephritidae). Z. angew. Entomol., $89,278-281$.

Katsoyannos B. I., Pittara I. S., 1983. Effect of size of artificial oviposition substrates and presence of natural host fruits on the selection of oviposition site by Dacus oleae. Entomol. exp. appl., $34,326-332$.

Klijnstra J. W., 1982. Perception of the oviposition deterrent pheromone in Pieris brassicae. Proc. 5th int. Symp. Insect-Plant Relationships, 145-151, Visser J. H., Minks A. K. ed., Pudoc Wageningen, $464 \mathrm{p}$.

Kogan M., 1976. The role of chemical factors in insect/plant relationships. Proc. XV Int. Congr. Entomol., Washington, 211-227.

Koslowski M. W., Lux S., Dmoch J., 1983. Oviposition behaviour and pod marking in the cabbage seed weevil, Ceuthorhynchus assimilis. Entomol. exp. appl., 34, 277-282.

Labeyrie V., 1960. Action de la présence de grains de haricot sur l'ovogenèse d'Acanthoscelides obtectus Say. C. R. Acad. Sci., Paris, Sér. D., 250, 2626-2628.

Labeyrie V., 1961. Obtention d'une souche astime chez Acanthoscelides obtectus Say. C. R. Soc. Biol., Paris, 155, 1366-1369.

Latheef M. A., Irwin R. D., 1979. Factors affecting oviposition of Pieris rapae on cabbage. Environ. Entomol., 8, 606-609.

Latheef M. A., Ortiz J. H., 1983a. The influence of companion herbs on egg distribution of the imported cabbageworm, Pieris rapae (Lepidoptera : Pieridae) on collard plants. Can. Entomol., 115, 1031-1038.

Latheef M. A., Ortiz J. H., $1983 b$. Influence of companion plants on oviposition of imported cabbageworm, Pieris rapae (Lepidoptera : Pieridae), and cabbagelooper Trichoplusia ni (Lepidoptera : Noctuidae), on collard plants. Can. Entomol., 115, 1529-1531.

Latheef M. A., Ortiz J. H., 1984. Influence of companion herbs on Phyllotreta cruciferae (Coleoptera: Chrysomelidae) on collard plants. J. econ. Entomol., 77, 80-82.

Latheeff M. A., Ortiz J. H., Sheikh A. Q., 1984. Influence of intercropping on Phyllotreta cruciferae (Coleoptera: Chrysomelidae) populations on collard plants. J. econ. Entomol., 77, 1180-1184.

Lerin J., 1980. Influence des substances allélochimiques des crucifères sur les insectes. Acta Oecol., Oecol. gener., 1, 215-235.

Leroi B., 1975. Influence d'une plante-hôte des larves (Apium graveolens $\mathrm{L}$.) sur la stimulation de la ponte et de la production ovarienne de Philophylla heraclei. C. R. Acad. Sci., Paris, Sér. D, 281 , 1015-1018.

Levinson H. Z., Haisch A., 1984. Optical and chemosensory stimuli involved in host recognition and oviposition of the cherry fly Rhagoletis cerasi L. Z. angew. Entomol., 97, 85-91.

Levinson H. Z., Levinson A. R., 1984. Botanical and chemical aspects of the olive tree with regards to fruit acceptance by Dacus oleae Gmelin and other frugivorous animals. Z. angew. Entomol., 98, 136-149.

Lundgren L., 1975. Natural plant chemicals acting as oviposition deterrents on cabbage butterflies (Pieris brassicae (L.), P. rapae (L.) and P. napi (L.)). Zool. Scripta, 4, 253-258.

Ma W. C., 1969. Some properties of gustation in the larva of Pieris brassicae. Entomol. exp. appl., 12, 584-590. 
Ma W. C., Schoonhoven L. M., 1973. Tarsal contact chemosensory hairs of the large white butterfly Pieris brassicae and their possible role in oviposition behaviour. Entomol. exp. appl., 16, 343-357.

Maguire L. A., 1984. Influence of surrounding plants on densities of Pieris rapae L. eggs and larvae (Lepidoptera : Pieridae) on collards. Environ. Entomol., 13, 464-468.

Mc Neil J. N., Quiring D. T., 1983. Evidence of an ovipositiondeterring pheromone in the alfafa blotch leafminer, Agromyza frontella Rondani (Diptera: Agromyzidae). Environ. Entomol., 12, 990-992.

Mehta R. C., Saxena K. N., 1970. Ovipositional responses of the cotton spotted bollworm Earias fabia (Lepidoptera : Noctuidae) in relation to its establishment on various plants. Entomol. exp. appl., 13, 10-20.

Messina J. F., Renwick J. A. A., 1985. Mechanism of egg recognition by the cowpea weevil Callosobruchus maculatus. Entomol. exp. appl., 37, 241-245.

Mitchell N. D., 1977. Differential host selection by Pieris brassicae (the large white butterfly) on Brassica oleracea subsp. oleracea (the wild cabbage). Entomol. exp. appl., 22, 208-219.

Mudd A., Corbet S. A., 1973. Mandibular gland secretion of larvae of the stored products pests Anagasta kuehniella, Ephestia cautella, Plodia interpunctella, and Ephestia elutella. Entomol. exp. appl., 16, 291-293.

Mumtaz M. M., Aliniazee M. T., 1983a. The oviposition-deterring pheromone in the western cherry fruit fly, Rhagoletis indifferens Curran (Dipt. : Tephritidae). 1. Biological properties. Z. angew. Entomol., 96, 83-93.

Mumtaz M. M., Aliniazee M. T., 1983 $b$. The oviposition-deterring pheromone in the western cherry fruit fly, Rhagoletis indifferens Curran (Dipt. : Tephritidae). 2. Chemical characterization and partial purification. Z. angew. Entomol., 96, 93-99.

Myers J. H., Monro J., Murray N., 1981. Egg clumping, host plant selection and population regulation in Cactoblastis cactorum (Lepidoptera). Oecologia, 51, 7-13.

Nair K. S., McEwen F. L., 1976. Host selection by the adult cabbage maggot, Hylemya brassicae (Diptera : Anthomyiidae) : effect of glucosinolates and common nutrients on oviposition. Can. Entomol., 108, 10, 1021-1030.

Neilson W. T. A., 1967. Development and mortality of the apple maggot Rhagoletis pomonella in crab apples. Can. Entomol., 99, 217-219.

Nordlund D. A., 1981. Semiochemicals : a review of the terminology, 13-28. In Nordlung D. A., Jones R. L., Lewis W. J. : «Semiochemicals: their role in pest control », Wiley Press New York, $306 \mathrm{p}$.

Den Otter C. J., Schuil H. A., Sander Van Oosten A., 1978. Reception of host plant odours and female sex pheromone in Adoxophyes orana (Lepidoptera : Tortricidae) : electrophysiology and morphology. Entomol. exp. appl., 24, 570-578.

Den Otter C. J., Behan M., Maes F. W., 1980. Single cell response in female Pieris brassicae (Lepidoptera : Pieridae) to plant volatiles and conspecific egg odours. J. Insect Physiol., 26, 465-472.

Panasiuk O., 1984. Response of Colorado potato beetles, Leptinotarsa decemlineata Say, to volatile components of tansy, Tanacetum vulgare. J. chem. Ecol., 10, 1325-1333.

Perrin R. M., Phillips M. L., 1978. Some effects of mixed cropping on the population dynamics of insect pests. Entomol. exp. appl., 24, 585-593.

Pesson P., 1980. A propos de l'instinct botanique des insectes: un aspect de la co-évolution des plantes et des insectes. Ann. Soc. entomol. Fr. (N.S.), 16, 435-452.

Philips P. A., Barnes M. M., 1975. Host race formation among sympatric apple, walnut and plum population of the codling moth, Laspeyresia pomonella. Ann. Entomol. Soc. Am., 68, 1053-1060.

Pierce H. D., Vernon R. S., Borden J. H., Oehlschlager A. C., 1978. Host selection by Hylemya antiqua (Meigen). Identification of three new attractants and oviposition stimulants. J. chem. Ecol., 4, 65-72.

Pouzat J., 1970. Rôle des organes sensoriels céphaliques dans l'ovogenèse et l'émission chez la bruche du haricot Acanthoscelides obtectus Say. Coll. int. C.N.R.S. ${ }^{\circ} 189,381-400$. Les stimuli externes sur la gamétogenèse des insectes, $422 \mathrm{p}$.
Pouzat J., 1976. Le comportement de ponte de la bruche du haricot en présence d'extrait de plante-hôte. Mise en évidence d'interactions gustatives et tactiles. C. R. Acad. Sci., Paris, Sér. D, 282, 19711974.

Pouzat J., 1978. Host plant chemosensory influence on oogenesis in the bean weevil, Acanthoscelides obtectus (Coleoptera: Bruchidae). Entomol. exp. appl., 24, 601-608.

Pouzat J., 1980. Insectes et "substances secondaires " des végétaux. In: «Les phéromones sexuelles et les médiateurs chimiques chez les insectes : utilisation en lutte intégrée $», 183-196$, Les colloques de l'INRA, 3, Colmar, 25-27 nov. 1980, $206 \mathrm{p}$.

Pouzat J., 1981. The role of sense organs in the relations between Bruchids and their host plants, 61-72. In V. Labeyrie : «The ecology of bruchids attacking legumes ", Junk publ., 233 p.

Prokopy R. J., 1968. Visual responses of apple maggot flies, Rhagoletis pomonella (Diptera: Tephritidae) : orchard studies. Entomol. exp. appl., 11, 403-422.

Prokopy R. J., 1972. Evidence for a marking pheromone deterring repeated oviposition in apple maggot flies. Environ. Entomol., 1, 326-332.

Prokopy R. J., 1977. Stimuli influencing trophic relations in Tephritidae. Coll. int. C.N.R.S. $\mathrm{n}^{\circ} 265,305-336$ : “ Comportement des insectes et milieu trophique », $493 \mathrm{p}$.

Prokopy R. J., 1981 $a$. Epideictic pheromones that influence spacing patterns of phytophagous insects, 181-213. In D. A. Nordlung, R. L. Jones, W. J. Lewis : «Semiochemicals: their role in pest control », Wiley Press New York, 306 p.

Prokopy R. J., $1981 b$. Oviposition-deterring pheromone system of apple maggot flies, 477-494. In E. R. Mitchell : « Management of insect pest with semiochemicals : Concept and Practice », Plenum Press, New York, $514 \mathrm{p}$.

Prokopy R. J., Boller E. F., 1971. Stimuli eliciting oviposition of European cherry fruit flies, Rhagoletis cerasi L. (Diptera: Tephritidae), into inanimate objects. Entomol. exp. appl., 14, 1-4.

Prokopy R. J., Haniotakis E. G., 1975. Responses of wild and laboratory-cultured Dacus oleae to host plant color. Ann. Entomol. Soc. Am., 68, 73-77.

Prokopy R. J., Haniotakis E. G., 1976. Host detection by wild and lab-cultured olives flies. Symp. Biol. Hung., 16, 209-214.

Prokopy R. J., Koyana J., 1982. Oviposition site partitioning in Dacus cucurbitae. Entomol. exp. appl., 31, 428-432.

Prokopy R. J., Owens E. D., 1983. Visual detection of plant by herbivorous insects. Annu. Rev. Entomol., 28, 337-364.

Prokopy R. J., Reissig W. H., Moericke V., 1976. Marking pheromones deterring repeated oviposition in Rhagoletis flies. Entomol. exp. appl., 20, 170-178.

Prokopy R. J., Ziegler J. R., Wong T. T. Y., 1978. Deterrence of repeated oviposition by fruit-marking pheromone in Ceratitis capitata (Diptera : Tephritidae). J. chem. Ecol., 4, 55-63.

Prokopy R. J., Malavasi A., Morgante J. S., 1982a. Ovipositiondeterring pheromone in Anastrepha fraterculus flies. $J$. chem. Ecol., 8, 763-771.

Prokopy R. J., Averill A. L., Bardinelli C. M., Bowdan E. S., Cooley S. S., Crnjar R. M., Dundulis E. A., Roitberg C. A., Spatcher P. J., Tumlinson J. H., Weeks B. L., 1982b. Site of production of an oviposition-deterring pheromone component in Rhagoletis pomonella flies. J. Insect Physiol, 28, 1-10.

Prokopy R. J., Averill A. L., Cooley S. S., Roitberg C. A., Kallet C., 1982c. Variation in host acceptance pattern in apple maggot flies. Proc. Sth int. Symp. Insect-Plant Relationships, 123-129, Visser J. H., Minks A. K. ed., Pudoc Wageningen, 464 p.

Prokopy R. J., Collier R. H., Finch S., 1983a. Visual detection of host plants by cabbage root flies. Entomol. exp. appl., 34, 85-89. Prokopy R. J., Collier R. H., Finch S., 1983 $b$. Leaf color used by cabbage root flies to distinguish among host plants. Science, 221, 190-192.

Prokopy R. J., McDonald P. T., Wong T. T. Y., 1984. Interpopulation variation among Ceratitis capitata flies in host acceptance pattern. Entomol. exp. appl., 35, 65-69.

Raina A. K., 1981. Deterrence of repeated oviposition in sorghum shootfly, Atherigona soccata. J. chem. Ecol., 7, 785-790.

Rausher M. D., 1979. Egg recognition : its advantage to a butterfly. Anim. Behav., 27, 1034-1040. 
Rausher M. D., Mackay D. A., Singer M. C., 1981. Pre- and postalighting host discrimination by Euphydryas editha butterflies : the behavioural mechanisms causing clumped distributions of egg clusters. Anim. Behav., 29, 1220-1228.

Reissig W. H., Fein B. L., Roelofs W. L., 1982. Field tests of synthetic apple volatiles as apple maggot (Dipt. : Tephritidae) attractants. Environ. Entomol., 11, 1294-1298.

Reissig W. H., Stanley B. H., Roelofs W. L., Schwarz M. R., 1985. Test of synthetic apple volatiles in traps as attractants for apple maggot flies (Diptera : Tephritidae) in commercial apple orchards. Environ. Entomol., 14, 55-59.

Remund U., Katsoyannos B. I., Boller E. F., 1980. Zur Eiverteilung der Kirschenfliege Rhagoletis cerasi L. (Dipt. : Tephritidae) im Freiland. Mitt. Schweiz. Entomol. Ges., 53, 401-405.

Renwick J. A. A., 1983. Nonpreference mechanisms : plant characteristics influencing behaviour, 199-213. In P. A. Hedin : «Plant resistance to insects ", A.C.S. symposium series, $\mathrm{n}^{\circ} 208,375 \mathrm{p}$.

Renwick J. A. A., Radke C. D., 1981. Host plant constituents as oviposition deterrents for the cabbage looper, Trichoplusia ni. Entomol. exp. appl., 30, 201-204.

Renwick J. A. A., Radke C. B., 1982. Activity of cabbage extracts in deterring oviposition by the cabbage looper Trichoplusia ni. Proc. Sth int. Symp. Insect-Plant Relationships, 139-143, Visser J. H., Minks A. K. ed., Pudoc Wageningen, 464 p.

Renwick J. A. A., Radke C. B., 1983. Chemical recognition of host plants for oviposition by the cabbage butterfly, Pieris rapae (Lepidoptera : Pieridae). Environ. Entomol., 12, 446-450.

Richardson C. H., 1925. The oviposition response of insects. USDA, Dep. Bull., 1324, 1-17.

Risch S. J., Andow D., Altieri M. A., 1983. Agroecosystem diversity and pest control : date, tentative conclusions, and new research directions. Environ. Entomol., 12, 625-629.

Robert P. C., 1965. Influence de la plante-hôte sur l'activité reproductrice de la teigne de la betterave Scrobipalpa ocellatella Boyd. (Lepidoptera, Gelechiidae). Proc. XII int. Congr. Entomol. London, 552-553.

Robert P. C., 1970. Action stimulante de la plante-hôte sur l'activité reproductrice chez la teigne de la betterave Scrobipalpa ocellatella Boyd. (Lepidoptera Gelechiidae). Coll. int. CNRS, $\mathrm{n}^{\circ} 189$, pp. 147-162. Influence des stimuli externes sur la gamétogenèse des insectes, $422 \mathrm{p}$.

Robert P. C., 1971. Der Einfluss der Wirtspflanzen und der Nichtwirtspflanzen auf Eibildung und Eiablage der Rübenmotte Scrobipalpa ocellatella Boyd. (Lepidoptera Gelechildae). Acta Phytopathol. Acad. Sci. Hung., 6, 235-241.

Robert P. C., 1976. Inhibitory action of chestnut-leaf extracts (Castanea sativa Mill.) on oviposition and oogenesis of the sugar beet moth, Scrobipalpa ocellatella Boyd. (Lepidoptera Gelechiidae). Symp. Biol. Hung., 16, 223-227.

Robert P. C., Blaisinger P., 1978. Role of non-host plant chemicals in the reproduction of an oligophagous insect : the sugar beet moth Scrobipalpa ocellatella (Lepidoptera : Gelechiidae). Entomol. exp. appl., 24, 632-636.

Robert P. C., Blaisinger P., Simonis M. T., 1977. Relations entre un insecte phytophage : la teigne de la betterave (Scrobipalpa ocellatella Boyd., Lepidopt. Gelechiidae) et sa plante-hôte. Fluctuations du comportement de ponte dans des populations naturelles en absence de signaux chimiques d'origine végétale. Coll. int. CNRS, $\mathrm{n}^{\circ} 265,269-283$. Comportement des insectes et milieu trophique, $493 \mathrm{p}$.

Roitberg B. D., Prokopy R. J., 1981. Experience required for pheromone recognition by the apple maggot fly. Nature, 292, 540-541.

Roitberg B. D., Propoky R. J., 1983. Host deprivation influence on reponse of Rhagoletis pomonella to its oviposition deterring pheromone. Physiol. Entomol., 8, 69-72.

Roitberg B. D., Prokopy R. J., 1984. Host discrimination by adult and larval European apple sawflies Hoplocampa testitudinea Klug (Hymenoptera : Tenthredinae). Environ. Entomol., 13, 1000-1003.

Roitberg B. D., Cairl R. S., Prokopy R. J., 1984. Oviposition deterring pheromone influences dispersal distance in tephritid fruit flies. Entomol. exp. appl., 35, 217-220.

Rothschild M., Schoonhoven L. M., 1977. Assessment of egg load by Pieris brassica' (Leptdoptera : Pieridae). Nature, 266, 5600, 352355 .
Röttger U., 1979a. Untersuchungen zur Wirtswahl der Rübenfliege Pegomya betae Curt. (Diptera, Anthomyïdae). I. Olfaktorische Orientierung zur Wirtspflanze. Z. angew. Entomol., 87, 337-348.

Röttger U., 1979b. Untersuchungen zür Wirtswahl der Rübenfliege Pegomya betae Curt. (Diptera, Anthomyiidae). II. Optische Orientierung zur Wirtspflanze. Z. angew. Entomol., 88, 97-107.

Sanders W., 1962. Das Verhalten der Mittelmeerfruchtfliege Ceratitis capitata Wied. bei der Eiablage. Z. Tierpsychol., 19, 1-28.

Sanders W., 1968. Die Eiablagehandlung der Mittelmeerfruchtfliege Ceratitis capitata Wied. Ihre Abhängigkeit von Grösse und Dichte der Früchte. Z. Tierpsychol., 25, 1-21.

Saringer G., 1976. Oviposition behaviour of Ceuthorrhynchus maculaalba Herbst. (Col. : Curculionidae). Symp. Biol. Hung., 16, 241-245.

Saxena K. N., Goyal S., 1978. Host-plant relations of the citrus butterfly Papilio demoleus L. : orientational and ovipositional responses. Entomol. exp. appl., 24, 1-10.

Saxena K. N., Basit A., 1982a. Inhibition of oviposition by volatiles of certain plants and chemicals in the leafhopper Amrasca devastans (Distant). J. chem. Ecol., 8, 329-338.

Saxena K. N., Basit A., 1982b. Interference with the establishment of the leaf hopper Amrasca devastans on its host plants by certain non-host plants. Proc. Sth int. Symp. Insect-Plant Relationships, 153-162, Visser J. H., Minks A. K. ed., Pudoc Wageningen, 464 p.

Schoonhoven L. M., 1968. Chemosensory bases of host plant selection. Annu. Rev. Entomol., 13, 115-136.

Schoonhoven L. M., 1981. Chemical mediators between plants and phytophagous insects, 31-50. In D. A. Nordlund, R. L. Jones, W. J. Lewis : "Semiochemicals : their role in pest control", Wiley New York, 306 p.

Schoonhoven L. M., 1982. Biological aspects of antifeedants. Entomol. exp. appl., 31, 57-69.

Schoonhoven L. M., 1983. The role of chemoreception in hostplant finding and oviposition in phytophagous Diptera, 258-267. In : R. Cavalloro : "Fruit flies of economic importance". Proc. CEC/IOBC intern. Sympos., Athens, Nov. 1982, A. A. Balkema, Rotterdam, $642 \mathrm{p}$.

Schoonhoven L. M., Sparnaay T., Van Wissen W., Meerman J., 1981. Seven-week persistence of an oviposition-deterrent pheromone. J. chem. Ecol., 7, 583-588.

Schurr K., 1970. Host plant interactions. Adv. Front. Plant Sci., $20,147-157$

Schurr K., Holdaway F. G., 1970. Olfactory responses of females Ostrinia nubilalis (Lepidoptera : Pyraustinae). Entomol. exp. appl., 13, 455-461

Schütte F., 1966. Beobachtungen zum Zug von Faltern der Gattung Pieris Schrk. Z. angew. Entomol., 58, 131-138.

Seo S. T., Tang C. S., Sanidad S., Takenaka T. H., 1983. Hawaiian fruit flies (Diptera: Tephritidae) : variation of index of infestation with benzyl isothiocyanate concentration and color of maturing papaya. J. econ. Entomol., 76, 535-538.

Shapiro A. M., 1981a. Egg-mimics of Streptanthus (Cruciferae) deter oviposition by Pieris sisymbrii (Lepidoptera : Pieridae). Oecologia, 48, 142-143.

Shapiro A. M., $1981 b$. The pierid red-egg syndrome. Am. Natur., 117, 276-294.

Sharma R. N., Joshi V., Zadu G., Bhosale A. S., Gupta A. S., Patwardhan S., Nanda B., 1981. Oviposition deterrence activity in some Lamiaceae plants against some insect pests. Z. Naturforsch., sect. C. Biosci., 36, 122-125.

Singer M. C., 1983. Determinants of multiple host use by a phytophagous insect population. Evolution, 37, 389-403.

Sparks M. R., 1973. Physical and chemical stimuli affecting oviposition preference of Manducta sexta (Lepidoptera: Sphingidae). Ann. Ent. Soc. Am., 66, 571-573.

Städler F., 1972. Über die Orientierung und das Wirtswahlverhalten der Mohrenfliege Psila rosae F. (Diptera: Psilidae). II. Imagines. Z. angew. Entomol., 70, 29-61.

Städler E., 1974. Host plant stimuli affecting oviposition behaviour of the eastern spruce budworm. Entomol. exp. appl., 17, 176-188.

Städler E., 1977. Host selection and chemoreception in the carrot rust fly Psila rosae F. (Dipt. Psilidae) extraction and isolation of 
oviposition stimulants and their perception by the female. Coll. int. C.N.R.S., $\mathrm{n}^{\circ} 265,357-372$. Comportement des insectes en milieu trophique, $493 \mathrm{p}$.

Städler E., 1978. Chemoreception of host plant chemicals by ovipositing females of Delia (Hylemia) brassicae. Entomol. exp. appl., $24,711-720$.

Städler E., Buser H. R., 1982. Oviposition stimulants for the carrot fly in the surface wax of carrot leaves. Proc. 5th Int. Symp. InsectPlant Relationships, 403-404, Visser J. H., Minks A. K. ed., Pudoc Wageningen, $464 \mathrm{p}$.

Stansly P. A., Cate J. R., 1984. Discrimination by ovipositing boll weevils (Coleoptera: Curculionidae) against previously infested Hampea (Malvaceae) flower buds. Environ. Entomol., 13, 13611365.

Stanton M. L., 1979. The role of chemotactile stimuli in the oviposition preference of Colias butterflies. Oecologia, 39, 79-91.

Stanton M. L., Cook R. E., 1984. Sources of intraspecific variation in the host plant seeking behavior of Colias butterflies. Oecologia, $61,265-270$.

Stockel J. P., 1981. Influence d'extraits aromatiques de grains de maïs sur l'activité reproductrice de Sitotroga cerealella Oliv. (Lepidoptera, Gelechiidae) en conditions de laboratoire. C. R. Acad. Sci. Paris, Sér. D, 292, 653-656.

Stockel J. P., Boidron J. N., 1981. Influence d'extraits aromatiques de grains de maïs sur l'activité reproductrice de l'alucite des céréales Sitotroga cerealella (Lepidoptera Gelechiidae) en conditions naturelles. C.R. Acad. Sci. Paris, Sér. D, 292, 343-346.

Sutherland O. R. W., Wearing C. H., Hutchins R. F. N., 1977. Production of $\alpha$-farnesene, an attractant and oviposition stimulant for codling moth, by developing fruit of ten varieties of apple. $J$. chem. Ecol., 3, 625-631.

Swift F. C., 1982. Field tests of visual and chemical lures for apple maggot flies. J. econ. Entomol., 75, 201-206.

Szentesi A., 1981. Pheromone-like substances affecting host related behaviour of larvae and adults in the dry bean weevil, Acanthoscelides obtectus. Entomol. exp. appl., 30, 219-226.

Szentesi A., Greany P. D., Chambers D. L., 1979. Oviposition behavior of laboratory-reared and wild Caribbean fruit flies (Anastrepha suspensa) (Diptera: Tephritidae). I. Selected chemical influences. Entomol. exp. appl., 26, 227-238.

Tabashnik B. E., Wheelock H., Rainbolt J. D., Watt W. B., 1981. Individual variation in oviposition preference in the butterfly, Colias eurytheme. Oecologia, 50, 225-230.

Tahvanainen J. O., Root R. B., 1972. The influence of vegetational diversity on the population ecology of a specialized herbivore, Phyllotreta cruciferae (Coleoptera : Chrysomelidae). Oecologia, 10, 321-346.

Terofal F., 1965. Zum Problem der Wirtspezifität bei Pieriden (Lep.). Mitt. münchner entomol. Ges., 55, 1-76.

Theunissen J., den Ouden H., 1980. Effects of intercropping with Spergula arvensis on pests of Brussels sprouts. Entomol. exp. appl., 27, 260-268.

Thibout E., Auger J., 1983. Substances chimiques secondaires des Allium déterminant le comportement de ponte de la teigne du poireau, Acrolepiopsis assectella Zell. Bull. Soc. entomol. Fr., 88, 359368 .

Thibout E., Auger J., Lecomte C., 1982. Host plant chemicals responsible for attraction and oviposition in Acrolepiopsis assectella. Proc. 5th int. Symp. Insect-Plant Relationships, 107-115, Visser J. H., Minks A. K. ed., Pudoc Wageningen, 464 p.

Thibout E., Anger J., Dakkouni M., 1985. Conservation à court terme de l'information chimique issue de la plante-hôte chez la teigne du poireau (Lépidoptère). C. R. Acad. Sci. Paris, Sér. III, 300, 489-492.

Thorsteinson A. J., 1960. Host selection in phytophagous insects. Annu. Rev. Entomol., 5, 193-218.

Tichenor L. H., Seigler D. S., 1980. Electroantennogram and oviposition responses of Manduca sexta to volatile components of tobacco and tomato. J. Insect Physiol., 26, 309-314.

Tingle F. C., Mitchell E. R., 1984. Aqueous extracts from indigenous plants as oviposition deterrents for Heliothis virescens F. $J$. chem. Ecol., 10, 101-113.

Traynier R. M. M., 1967a. Effect of host plant odour on the beha- viour of the adult cabbage root fly Erioischia brassicae. Entomol. exp. appl., 10, 321-328.

Traynier R. M. M., $1967 \mathrm{~b}$. Stimulation of oviposition by the cabbage root fly Erioischia brassicae. Entomol. exp. appl., 10, 401-412.

Traynier R. M. M., 1979. Long-term changes in the oviposition behaviour of the cabbage butterfly, Pieris rapae, induced by contact with plants. Physiol. Entomol., 4, 87-96.

Tukahirwa E. M., Coaker T. H., 1982. Effect of mixed cropping on some insect pests of brassicas ; reduced Brevicoryne brassicae infestations and influences on epigeal predators and the distribution of oviposition behaviour in Delia brassicae. Entomol. exp. appl., $32,129-140$

Uvah I. I. I., Coaker T. H., 1984. Effect of mixed cropping on some insect pests of carrots and onions. Entomol. exp. appl., 36, 159-167.

Van Lenteren J. C., 1981. Host discrimination by parasitoids, 153179. In : Nordlund D. A., Jones R. L., Lewis W. J. : "Semiochemicals their role in pest control"'. Wiley Press New York, $306 \mathrm{p}$.

Vernon R. S., Borden J. H., Pierce H. D., Oehlschlager A. C., 1977. Host selection by Hylemya antiqua. Laboratory bioassay and methods of obtaining host volatiles. J. chem. Ecol., 3, 359-368.

Vernon R. S., Pierce H. D., Borden J. H., Oehlschlager A. C., 1978. Host selection by Hylemya antiqua : identification of oviposition stimulants based on proposed active thioalkane moieties. Environ. Entomol., 7, 728-731.

Verschäffelt E., 1910. The cause determining the selection of food in some herbivorous insects. Proc. Acad. Sci., Amsterdam, 13, 536542.

Visser J. H., 1983. Differential sensory perceptions of plant compounds by insects, 215-230. In: P. A. Hedin : "Plant resistance to insects”. A.C.S. symposium serie $\mathrm{n}^{\circ} 208,375 \mathrm{p}$.

Wallbank B. E., Wheatley G. A., 1979. Some responses of cabbage root fly (Delia brassicae) to allyl isothiocyanate and other volatile constituents of crucifers. Ann. appl. Biol., 91, 1-12.

Wasserman S. S., 1981. Host-induced preferences and oviposition markers in the cowpea weevil, Callosobruchus maculatus. Ann. Entomol. Soc. Am., 74, 242-245.

Wiens M. N., Rahe J. E., Vernon R. S., McLean J. A., 1978. Ovipositional deterrents for Hylemya antiqua in hydrated seeds of Phaseolus vulgaris. Environ. Entomol., 7, 165-167.

Wiklund C., 1974. Oviposition preferences in Papilio machaon in relation to the host plants of the larvae. Entomol. exp. appl., 17, 189-198.

Wiklund C., 1981. Generalist vs. specialist oviposition behaviour in Papilio machaon (Lepidoptera) and functional aspects on the hierarchy of oviposition preferences. Oikos, 36, 163-170.

Wiklund C., 1982. Generalist versus specialist utilization of host plants among butterflies. Proc. 5th int. Symp. Insect-Plant Relationships, 181-191, Visser J. H., Minks A. K. ed., Pudoc Wageningen, $464 \mathrm{p}$.

Wiklund C., 1984. Egg-laying patterns in butterflies in relation to their phenology and the visual apparency and abundance of their host plants. Oecologia, 63, 23-29.

Williams K. S., 1983. The coevolution of Euphydryas chalcedona butterflies and their larval host plants. III. Oviposition behavior and host plant quality. Oecologia, 56, 336-340.

Williams K. S., Gilbert L. E., 1981. Insects as selective agents on plant vegetative morphology : egg mimicry reduces egg-laying by butterflies. Science, 212, 467-469.

Wolfson J. L., 1980. Oviposition response of Pieris rapae to environmentally induced variation in Brassica nigra. Entomol. exp. appl., 27, 223-232.

Yamamoto R. T., Fraenkel G., 1960. The physiological basis for the selection of plants for egg-laying in the tobacco hornworm, Protoparce sexta Johan. Proc. XI. int. Congr. entomol., Wien, 3, 127133.

Yamamoto R. T., Jenkins R. Y., McClushy R. K., 1969. Factors determining the selection of plants for oviposition by the tobacco hornworm Manduca sexta. Entomol. exp. appl., 12, 504-508.

Zohren E., 1968. Laboruntersuchungen zu Massenanzucht, Lebensweise, Eiablage und Eiablageverhalten der Kohlfliege, Chortophila brassicae Bouché (Diptera: Anthomyiidae). Z. angew. Entomol., $62,139-188$ 
Zwölfer H., Preiss M., 1983. Host selection and oviposition behaviour in West-European ecotypes of Rhinocyllus conicus Froel. (Col., Curculionidae). Z. angew. Entomol., 95, 113-122.
Zwölfer H., Harris P., 1984. Biology and host specificity of Rhinocyllus conicus Froel. (Col., Curculionidae) a successful agent for biocontrol of the thistle, Carduus nutans L. Z. angew. Entomol., 